
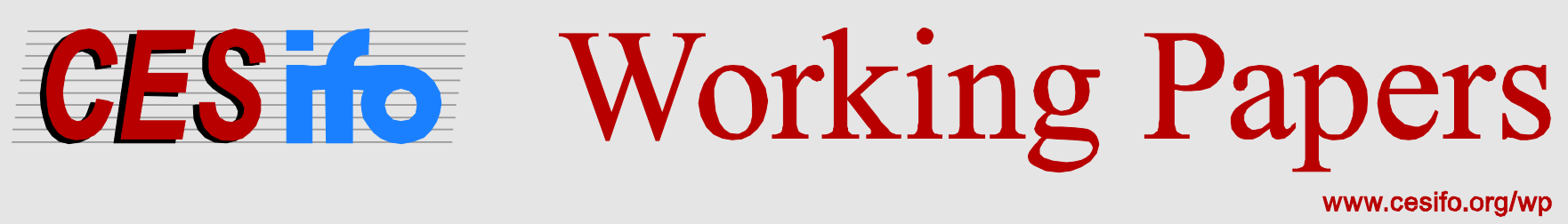

\title{
Dynamic Resource Management under Weak Property Rights: A Tale of Thieves and Trespassers
}

\author{
Mauricio Rodriguez \\ Sjak Smulders
}

\author{
CESIFO WORKING PAPER NO. 6019 \\ CATEGORY 9: RESOURCE AND ENVIRONMENT ECONOMICS \\ ORIGINAL VERSION: JULY 2016 \\ THIS VERSION: JANUARY 2021
}

An electronic version of the paper may be downloaded

- from the SSRN website:

- from the RePEc website:

- from the CESifo website:

wWW.SSRN.com

www.RePEc.org

www.CESifo-group.org/wp 


\title{
Dynamic Resource Management under Weak Property Rights: A Tale of Thieves and Trespassers
}

\begin{abstract}
We study non-renewable resource extraction when institutions weakly protect the resource owner's property rights. First, weak wealth protection exposes the stock in the ground to trespassing. Second, weak income protection exposes revenues from extraction to theft. In our dynamic framework with strategic interactions, the strength of wealth and income protection evolves over time. The weak protection of wealth results in excessive depletion due to the common pool externality. Anticipated changes in institutional strength further distort depletion. A resource user (i.e., owner or trespasser) is less rapacious when she anticipates favorable institutional changes. However, a given change in institutional strength may be favorable for some but detrimental for another resource user and can therefore either mitigate or exacerbate the common pool externality. Our results indicate that unstable institutions limit the benefits derived from resource ownership and thus constitute a challenge to the efficient management of non-renewable resource riches in weakly institutionalized economies.
\end{abstract}

JEL-Codes: K420, P480, Q320, Q380.

Keywords: depletion, institutions, non-renewable resources, weak property rights.

\author{
Mauricio Rodriguez \\ Department of Economics \\ Uniiversidad del Rosario \\ Colombia - 111711 Bogotá \\ mauricio.rodriguez@urosario.edu.co
}

\author{
Sjak Smulders \\ Department of Economics, TSC \\ Tilburg University \\ P.O. Box 90153 \\ The Netherlands - 5000 LE Tilburg \\ j.a.smulders@tilburguniversity.edu
}

January 14, 2021

We thank Geir Asheim, Inge van den Bijgaart, Snorre Kverndokk, Hiro Sakamoto, Daniel Spiro, Aart de Zeeuw, Amos Zemel, and the seminar participants at the Graduate Institute Geneva, ISCTE-IUL, University of Gothenburg, University of Oslo, University of Porto, Universidad del Rosario, Tilburg Sustainability Center, ORCOS workshop, SURED, and EAERE, for helpful comments. 


\section{Introduction}

The problem of weak property rights is pervasive across economic settings. Such is the case of the management of non-renewable resources, where property rights have been for long central in the discussion of how to secure the optimal use of these resources. In this context, a weak protection of property rights is typically associated with a problem of common access to the stock of the resource, and 'weak property rights' are taken as synonymous with resource over-use.

However, the management of non-renewable resources can also be affected by insecure rights over the revenues from exploiting the resource. This second manifestation of weak property rights reduces the effective revenues that can be obtained from exploiting resource, and thus may end up distorting the trade-offs that govern the resource management decisions.

Weakly institutionalized economies struggle to obtain the most out of their resource riches. Imperfect protection of both resource wealth and income play a role in this resource curse. Take the case of gold mining in Latin America (Banchirigah, 2008, Hilson, 2002, Hilson \& Potter, 2003). The weak protection of rights over the resource wealth in the ground (i.e., mining rights) has resulted in widespread illegal mining across the region: ". . . about $28 \%$ of gold mined in Peru, $30 \%$ of gold mined in Bolivia, $77 \%$ of gold mined in Ecuador, $80 \%$ of gold mined in Colombia and $80-90 \%$ of Venezuelan gold is produced illegally" (Wagner.2016, p. vi). Besides the weak protection of stocks in the ground, legal and illegal miners in the region are significantly exposed to effective income losses due to extortion, theft, and violence (Channing \& Clough, 2017, Idrobo, Mejía, \& Tribin, 2014, Rosales, 2019.

A notorious example is the situation of the Orinoco Mining Arc in Venezuela. This vast mineral rich area was designated by the government as a special zone for the development mining projects, in the hopes to find substitutes for its dwindling oil revenues (Rosales, 2017). However, the dire economic and political situation that the country has experienced in the last couple of decades, has been accompanied by a weak rule of law (Bull \& Rosales, 2020). As consequence, illegal mining operations and criminal organizations fighting for the control of mining sites are ubiquitous in the Orinoco Mining Arc (Bull \& Rosales, 2020: Soud, 2019). According to a recent report by the Office of the United Nations High Commissioner for Human Rights, this has exposed miners to "a pattern of labour exploitation whereby miners are forced to give up a large percentage of the gold they extract to the various actors who control the mines. They are generally required to pay about 10 to 20 per cent of the ore mined to the criminal groups or armed elements in order to work." (OHCHR, 2020, p. 9) 1

Apart from featuring the exploitation of a non-renewable resource under weak property rights, where both the resource wealth in the ground and the income from extraction are imperfectly protected, the case of the Orinoco Mining Arc also offers a view on the dynamic nature of institutional settings. At the beginning of the 2000s, gold mining in the area was largely undertaken by foreign firms in a system of concessions. This came to an end with the nationalization of the mining sector in 2011, which ultimately led to the abandonment of large-scale projects by foreign firms (Rosales, 2017;

\footnotetext{
${ }^{1}$ Similar patterns of revenue insecurity have been identified in Mexico and Colombia where drug trafficking organizations are reported to charge hefty fees to allow miners to operate in areas controlled by these organizations (Fox 2012). Similarly, prompted by the use of gold in money laundering, these criminal organizations also appear to be actively engaged in the theft of gold shipments (Pachico 2012).
} 
Wilburn, 2014). With the continuous deterioration of the rule of law in the country, the vacuum left by the foreign firms was filled by illegal mining and the control of economic activity in the mining areas fell in the hands of illegal armed organizations ${ }^{2}$ This combination of factors resulted in a weakening of the rights over the stocks in the ground and the revenues above it ( $\mathrm{OHCHR}, 2020)$.

Against this backdrop, this paper contributes to the literature by studying the exploitation of a non-renewable resource in a setting with institutions that evolve over time and affect different interest groups. We put forward a model of non-renewable resource management featuring the imperfect protection of wealth and income. We model the imperfect protection of wealth by allowing extraction from the resource stock by agents other than the 'rightful' owner of the resource. The imperfect protection of income is introduced as a game of forceful appropriation of revenues from exploiting the resource (i.e., agents other than those generating the revenues from extraction can appropriate these revenues). Given our focus on non-renewable resources, our setup is dynamic. This dynamic setup is not only a convenient representation of a non-renewable resource management problem, but also lends itself to incorporate stochastic institutional dynamics. We thus not only model currently weak institutions, but also their 'instability' and the expectations this creates for resource users. This allows us to identify how the static and dynamic aspects of weakly institutionalized settings ultimately affect the ability of the resource owner to benefit from the resource. Our setting is also designed to allow for conflicts of interests. In an uncertain and unstable institutional environment, institutional changes affect rivaling resource managers differently, creating winners and losers. The anticipation of losses and gains unleashes a strategic interaction between different players that is different in nature from the conventionally studied common pool externality. The conflict of interest in our model arises because we separate wealth protection from income protection, and allow both dimensions to change non-simultaneously and in different intensity.

The problem of weak property rights in resource management has been commonly approached abstracting from institutional dynamics, and focusing on the wealth dimension. Under such approach, the imperfect protection of wealth results in a common pool problem and inefficiency arises in the form of excessive use of the resource (e.g., Copeland \& Taylor, 2009: Hardin, 1968: Van Long, 2011: Ostrom, 2008). This literature finds that resource use is excessive in the dynamic market equilibrium. Yet, in the presence of other imperfections, perfect wealth protection may not be necessary to attain optimal resource use. In a recent contribution, Croutzet and Lasserre (2017) show that the exclusive control over the stock of a renewable resource is not necessary to ensure optimal outcomes when resource use is distorted by market power.

As argued above, weak protection of revenues from resource exploitation may interact with the common access problem. Following this line of reasoning, Hotte, McFerrin, and Wills (2013) study a static production problem in which both the input and output are imperfectly protected. As an application of the second best theory (Lipsey \& Lancaster. 1956), their results indicate that in the presence of both sources of imperfection, resource use can be too high or too low relative to the social optimum. In particular, the weak protection of inputs will lead to excessive use, and hence excessive production, while the weak protection of output discourages productive efforts and acts in an opposite direction. Thus, optimal resource use may be attained even if inputs are imperfectly

\footnotetext{
${ }^{2}$ According to the Worldwide Governance Indicators, between 2000 and 2018 Venezuela dropped from the 25th percentile to the absolute bottom in the rule of law ranking (https://databank.worldbank.org/source/worldwide-governance-indicators).
} 
protected.

From the static approach in Hotte et al. (2013), we borrow the labels of trespassing and theft, but translate them to a dynamic setting in which stocks and flows, respectively, are weakly protected. The manifestation of imperfect wealth protection (i.e., imperfect protection of inputs in their model) is considered trespassing, and the manifestation of imperfect income protection (i.e., imperfect protection of output) is labeled as theft. Our model features three distinct, forward-looking, agents that interact strategically: owner, trespasser, and thief. The owner and the trespasser have access to a common stock of a non-renewable resource, from which they can extract and derive income flows. The main difference between these agents is that the trespasser effectively faces a lower productivity, which is associated to the cost of circumventing the system of property rights. Better protection of wealth translates into lower productivity for the trespasser; under perfect wealth protection the effective productivity of the trespasser is zero and there is no trespassing. The thief can forcefully appropriate income from the owner and the trespasser. Stealing requires a costly effort by the thief, and both the owner and the trespasser can exert costly protective efforts to protect their own income against theft. This means that under imperfect income protection the owner and trespasser effectively forgo a fraction of the potential revenues from extraction. Part of their revenues are captured by the thief, and part dissipates in the form of unproductive protective effort. Better income protection implies that the appropriation effort by the thief is relatively less productive than the protective efforts by the owner an the trespasser; under perfect income protection theft effort is absolutely unproductive, and there is no theft.

We use this framework to analyze the extent to which the evolution of the institutional setting limits the possibilities to get maximum benefits out of exploiting the resource wealth. Our results are particularly relevant to understand the management of non-renewable resource riches in contexts weak institutions and fluid political situations.

When, due to imperfect wealth protection, owner and trespasser have access to the same resource, we expect a common pool externality: the presence of other extractors reduces the control that each extractor expects to have over future stocks and thus lowers the incentive to invest in resource conservation. Excessive extraction can be offset if both agents have strong reasons to expect higher returns from future extraction. Anticipated improvements in future institutions may provide the reason. We find that under imperfect wealth protection, institutional dynamics are necessary to offset the excessive use of the resource caused by the common pool externality. When the owner and the trespasser anticipate favorable institutional changes, they have incentives to preserve the resource for future extraction under more favorable circumstances, which then results in more conservative extraction. The challenge here is that what constitutes a favorable institutional change is actually agent-specific. For instance, the owner benefits from stronger wealth protection, but the opposite is true for the trespasser. This asymmetric effect can result in excessive resource use when a strengthening of wealth protection is anticipated. In the case of theft, a stronger income protection is favorable both for the owner and the trespasser. If income protection is anticipated to become stronger, total resource use is relatively more conservative. If stronger income protection is anticipated, the owner and the trespasser have higher incentives to preserve the resource to extract it when it is less exposed to theft.

This document is organized in five sections including this introduction. Section 2 , presents the set 
up of the non-renewable resource management model with institutional dynamics along the wealth and income dimension. Section 3 , is devoted to the analytical solution of the model and the discussion of the main results. The solution strategy is presented there is general in the sense that that it applies to different configurations of institutional dynamics. Section 4 presents a numerical example of the model based on a path of deteriorating institutions, and discusses the example through the lenses of analytical results. Finally, Section 5 is devoted to the concluding remarks.

\section{The model}

Our setup consists of a continuous time infinite horizon model with three forward-looking agents: owner $(i)$, trespasser $(j)$, and thief $(k)$. The owner is initially endowed with the rights to exploit a stock $S_{0}>0$ of a non-renewable resource and can derive revenues from exploiting it. The protection of property rights is defined for each of the two types of property in the model: wealth (stock in the ground) and income (revenues from exploitation). When wealth is imperfectly protected the owner does not have exclusive access to the stock of the resource, and the trespasser can exploit it. When income is imperfectly protected the thief can appropriate a fraction of the owner's and trespasser's revenues from extraction. In the following we describe how institutional strength affects trespassing and theft, and what it entails for the strategic interactions between the three agents.

Wealth protection and trespassing Under imperfect wealth protection both the owner and the trespasser have access to the stock of the resource, and simultaneously decide how much of the resource to extract in a non-cooperative manner. Each agent has access to the same, income-generation technology from extraction. By extracting $R$ units of the resource, an agent gets a gross income flow of $Y\left(R_{s}\right)=\theta(\theta-1)^{-1} R_{s}^{1-\frac{1}{\theta}}$ with $\theta \in(1,2) 3^{3}$ Additionally, the trespasser needs to exert effort to elude law enforcement. This effort costs $T \geq 0$, which we assume is proportional to the trespasser's gross income. Specifically, $T=W Y\left(R_{j}\right)$, where $W \in[0,1]$ represents the institutional strength in terms of wealth protection. This cost function implies that it is costlier to elude law enforcement if the scale of the illegal operation is larger and if wealth protection is stronger.

Extraction depletes the resource over time, and the remaining stock $S(t)$ evolves according to $\partial S(t) / \partial t \equiv \dot{S}(t)=-R_{i}(t)-R_{j}(t)$, where $R_{s}$ denotes extraction by agent $s$; given that the resource is non-renewable, cumulative extraction is constrained by the remaining stock $\int_{t}^{\infty}\left(R_{i}(v)+R_{j}(v)\right) d v \leq$ $S(t) !$

Income protection and theft Under imperfect income protection, the thief can appropriate a fraction of the owner's and trespasser's net income $\left(Y\left(R_{i}\right)\right.$ and $\left.(1-W) Y\left(R_{j}\right)\right)$ by force. The fraction of agent $s^{\prime}$ income appropriated by the thief is endogenously determined by a ratio contest success

\footnotetext{
${ }^{3}$ The upper bound for $\theta$ guarantees the existence of an equilibrium in linear strategies in the trespassing game.

${ }^{4}$ Instead of a 'common stock' problem, trespassing could be introduced as 'common market' problem. In that case, trespassing would reduce the owner's marginal, market, return to extraction. Yet, the two approaches are equivalent. Trespassing increases depletion in the "common stock" approach, this would result in an increased scarcity rent of the resource and hence a lower effective return from extraction.
} 
function (Hirshleifer. 1989) of the form

$$
l\left(e_{s}, e_{k s}\right)=\frac{(1-L) e_{k s}}{L e_{s}+(1-L) e_{k s}}
$$

$e_{k s}$ denotes the appropriation effort by the thief, and $e_{s}$ is the protective effort by $s \in\{i, j\}$. We assume that net income is perfectly observable to all parties and that protection and appropriation efforts are simultaneously chosen. The relative efficiency of the protective effort, $e_{s}$, depends on the income-specific institutional strength, $L \in[0,1]$; for simplicity we assume that the strength of income protection applies equally to owner's and trespasser's income. Appropriation and protection efforts come at a constant unit $\operatorname{cost} \varepsilon>0$ for the corresponding agent ${ }^{5}$

Institutional dynamics Institutional strength can evolve along each of the two dimensions of property: wealth and income. We assume that institutional strength can take one of two values in each dimension, $W \in\left\{\omega_{0}, \omega_{1}\right\}$ and $L \in\left\{\lambda_{0}, \lambda_{1}\right\}$, where the initial states of wealth and income protection are given by $W(0)=\omega_{0}$ and $L(0)=\lambda_{0}$. Institutional dynamics manifest as regime shifts. In case of evolution in the wealth dimension, $W$ shifts to $\omega_{1}$, and in case of evolution in the income dimension $L$ becomes shifts to $\lambda_{1}$. There is at most one shift per dimension, and hence the sub-index 1 , in $\omega_{1}$ and $\lambda_{1}$, denotes the absorptive state of the respective institutional dimension: once $W=\omega_{1}$ it remains there, the same occurs when $L=\lambda_{1}$. In their initial states $\left(\omega_{0}\right.$ and $\left.\lambda_{0}\right)$ both $W$ and $L$ are bound to shift. These shifts do not necessarily occur at the same time, the precise timing of a shift is ex-ante, unknown, but its likelihood is common knowledge.

The institutional space $I$ consists of four possible regimes: $I=\{00,10,01,11\}$; we use the notation $x y$ to denote a regime in $I$ where the state of wealth protection is $W=\omega_{x}$ and the state of income protection is $L=\lambda_{y}$. The institutional path is such that economy starts in regime 00 and eventually reaches regime 11. There are 3 possible paths connecting regime 00 to regime 11: the economy can go directly from 00 to 11 or, it can transition either through regime 01 or 10. We follow Sakamoto (2014) to model the likelihood of a regime shift when multiple regime paths are possible, and model the evolution of institutional strength from regime 00 to regime 11 as governed by two types of parameters: i) the speed of institutional change, given by $\pi>0$; ii) conditional on regime shift, how likely is the change to occur along the wealth dimension or the income dimension, and this is given by the probabilities $p \in[0,1]$ and $q \in[0,1]$, respectively. This produces a flow probability (hazard) of $W$ shifting from $\omega_{0}$ to $\omega_{1}$ of $\pi p$, and a flow probability of $L$ shifting from $\lambda_{0}$ to $\lambda_{1}$ of $\pi q$. Wealth and income shifts are independent. Hence, the overall flow probability of a regime shift occurring while the economy is in the initial regime 00 is $\pi(p+q-p q)$.

Objective and equilibrium concept Each agent seeks to maximize the Net Present Value (NPV) of revenues, using the exogenous rate $r>0$ as a discount, and taking the expected evolution of the institutional setting as given. We focus on Markovian strategies, and rely on the Feedback Nash Equilibrium as equilibrium concept. The equilibrium of the trespassing game is based on linear strategies, where the extraction by each agent is set to be a linear function of the remaining stock.

\footnotetext{
${ }^{5}$ Note that this setup ensures that the owner-thief and trespasser-thief contests are independent, and there are no spillovers.
} 


\section{Equilibrium}

As a benchmark we present the case with perfect protection and static institutions, i.e., $W=1$ and $L=1 \forall t$. Then, we present a full characterization of extraction decisions, and protection and appropriation efforts in a general regime, $x y$. Finally, we use this characterization to describe each of the four possible regimes in the institutional space $I$, and solve the equilibrium of the model by moving backwards from regime 11 to regime 00 .

\subsection{Benchmark: Perfect protection}

As a benchmark we use the setting under which the resource owner can benefit the most from using the resource. This setting without distortions requires perfect protection of income and wealth, and no institutional dynamics such that there never is trespassing nor theft: $W=1$ and $L=1 \forall t$. Under perfect protection, the owner's problem is given by the following Hamilton-Jacobi-Bellman (HJB) equation:

$$
r V_{i}(t)=\max _{R_{i}}\left\{\frac{R_{i}(t)^{1-\frac{1}{\theta}}}{1-\frac{1}{\theta}}-\frac{\partial V_{i}(t)}{\partial S(t)} R_{i}(t)\right\} .
$$

The solution of this problem gives the optimal depletion rate from the owner's perspective

$$
D^{*} \equiv \frac{R_{i}^{*}(t)}{S(t)}=\theta r,
$$

and the optimal Net Present Value (NPV) of the remaining stock for the owner:

$$
V_{i}^{*}(S(t))=\frac{S(t)^{1-\frac{1}{\theta}}}{\left(1-\frac{1}{\theta}\right)(\theta r)^{\frac{1}{\theta}}} .
$$

\subsection{General regime, $x y$}

Relative to the perfect protection benchmark, the setup for a general regime $x y$ representing any of the regimes in the institutional space $I$ requires three adjustments: first, incorporate the costs associated to theft, which are the sum of revenues lost to theft and the cost of the protective effort; second, include the trespasser's extraction; third, account for all the possible future regimes in the continuation value.

The problem of the owner $(i)$ is to choose her own extraction of the resource $\left(R_{i}\right)$, as well as the protective effort against theft $\left(e_{i}\right)$, to maximize the NPV revenues net of theft costs. Taking into account the three adjustments required, the problem of the owner in regime $x y$ is represented by the following HJB equation

$$
r V_{i, x y}=\max _{\left\{R_{i}, e_{i}\right\}}\left\{\left(1-l\left(e_{i}, e_{k i}\right)\right) \frac{R_{i}^{1-\frac{1}{\theta}}}{1-\frac{1}{\theta}}-\varepsilon e_{i}-\frac{\partial V_{i, x y}}{\partial S}\left(R_{i}+R_{j}\right)+\pi_{x y}\left(\mathbf{E}_{x y}\left[V_{i}\right]-V_{i, x y}\right)\right\} ;
$$

$\mathbf{E}_{x y}\left[V_{i}\right]-V_{i, x y}$ is the expected gain of departing regime $x y$ conditional on departure, and $\pi_{x y}$ is the 
flow probability of departing this regime. For instance, in the initial regime, the expected gain conditional on departure is $\left(p(1-q) V_{i, 10}+q(1-p) V_{i, 01}+p q V_{i, 11}\right)(p+q-p q)^{-1}-V_{i, 00}$, and the flow probability of a regime shift is $\pi_{00}=\pi(p+q-p q)$.

The trespasser, as the owner, chooses the level of extraction $\left(R_{j}\right)$ and protective effort against theft $\left(e_{j}\right)$ to maximize the NPV of revenues net of trespassing and theft costs. This problem is represented by the following the HJB

$$
r V_{j, x y}=\max _{\left\{R_{j}, e_{j}\right\}}\left\{\left(1-l\left(e_{j}, e_{k j}\right)\right)\left(1-\omega_{x}\right) \frac{R_{j}^{1-\frac{1}{\theta}}}{1-\frac{1}{\theta}}-\varepsilon e_{j}-\frac{\partial V_{j, x y}}{\partial S}\left(R_{i}+R_{j}\right)+\pi_{x y}\left(\mathbf{E}_{x y}\left[V_{j}\right]-V_{j, x y}\right)\right\},
$$

with $l$ (.) given by 11 .

Finally, the problem of the thief is to choose the appropriation effort, $e_{k i}$ and $e_{k j}$, that maximize the NPV of appropriated revenues net of the effort costs:

$$
\begin{gathered}
r V_{k, x y}=\max _{\left\{e_{k i}, e_{k j}\right\}}\left\{l\left(e_{i}, e_{k i}\right) \frac{R_{i}^{1-\frac{1}{\theta}}}{1-\frac{1}{\theta}}+l\left(e_{j}, e_{k j}\right)\left(1-\omega_{x}\right) \frac{R_{j}^{1-\frac{1}{\theta}}}{1-\frac{1}{\theta}}-\varepsilon\left(e_{k i}+e_{k j}\right)\right. \\
\left.-\frac{\partial V_{k, x y}}{\partial S}\left(R_{i}+R_{j}\right)+\pi_{x y}\left(\mathbf{E}_{x y}\left[V_{k}\right]-V_{k, x y}\right)\right\} .
\end{gathered}
$$

Equilibrium: theft We start by solving the independent, owner-thief and trespasser-thief, theft games. If $\lambda_{y}=1$, income protection is perfect, there is no theft contest and the owner and the trespasser retain all the revenues from extraction. If $\lambda_{y}<1$, the appropriation and protection efforts in the owner-thief game are determined by the following first order conditions (FOC) for the owner and the thief, respectively:

$$
\begin{gathered}
\frac{\left(1-\lambda_{y}\right) \lambda e_{k i}(t)}{\left(\lambda_{y} e_{i}(t)+\left(1-\lambda_{y}\right) e_{k i}(t)\right)^{2}} \frac{R_{i}(t)^{1-\frac{1}{\theta}}}{1-\frac{1}{\theta}}-\varepsilon=0, \\
\frac{\left(1-\lambda_{y}\right) \lambda e_{i}(t)}{\left(\lambda_{y} e_{i}(t)+\left(1-\lambda_{y}\right) e_{k i}(t)\right)^{2}} \frac{R_{i}(t)^{1-\frac{1}{\theta}}}{1-\frac{1}{\theta}}-\varepsilon=0 .
\end{gathered}
$$

Solving for $e_{i}$ and $e_{k i}$, we obtain that the equilibrium levels of effort are increasing in the owner's revenues from extraction, and given by

$$
e_{i, x y}(t)=e_{k i, x y}(t)=\left(1-\lambda_{y}\right) \lambda_{y} \frac{R_{i, x y}(t)^{1-\frac{1}{\theta}}}{\left(1-\frac{1}{\theta}\right) \varepsilon} .
$$

Back into $\sqrt{1}$, this implies that the owner retains a fraction $\lambda_{y}$ of her revenues: $l\left(e_{i, x y}, e_{k i, x y}\right)=1-\lambda_{y}$. Using this, we obtain the owner's instantaneous flow of revenues net of stolen revenue and the cost of protection: 


$$
\left(1-l\left(e_{i}(t), e_{k i}(t)\right)\right) \frac{R_{i}(t)^{1-\frac{1}{\theta}}}{1-\frac{1}{\theta}}-\varepsilon e_{i}(t)=\lambda_{y} \frac{R_{i}(t)^{1-\frac{1}{\theta}}}{1-\frac{1}{\theta}}-\varepsilon e_{i, x y}(t)=\lambda_{y}^{2} \frac{R_{i}(t)^{1-\frac{1}{\theta}}}{1-\frac{1}{\theta}} .
$$

The net gain for the thief, i.e., the stolen revenue net of the cost of appropriation is

$$
\left(1-\lambda_{y}\right)^{2} \frac{R_{i}(t)^{1-\frac{1}{\theta}}}{1-\frac{1}{\theta}}
$$

Using the last two expressions one can see that waste is inherent to the theft game. The distribution of revenues between the owner and the thief requires exerting nonproductive, yet costly, efforts $e_{i}$ and $e_{k i}$. The deadweight loss caused by this inefficient distribution mechanism is a fraction $2 \lambda(1-\lambda)$ of the owner's revenues. This fraction is maximal when the theft game is most equalized: $\lambda=1 / 2$

The trespasser-thief game is essentially the same as the owner-thief game. Following the previous derivations, we obtain that the trespasser's instantaneous flow of revenues net of the cost of trespassing, theft, and the cost of protection is

$$
\left(1-\omega_{x}\right) \lambda_{y}^{2} \frac{R_{j}(t)^{1-\frac{1}{\theta}}}{1-\frac{1}{\theta}}
$$

and the net gain for the thief is

$$
\left(1-\lambda_{y}\right)^{2}\left(1-\omega_{x}\right) \frac{R_{j}(t)^{1-\frac{1}{\theta}}}{1-\frac{1}{\theta}} .
$$

Again, the theft contest results in a deadweight loss equivalent to a fraction $2 \lambda(1-\lambda)$ of the trespasser's revenues net of trespassing costs.

Equilibrium: trespassing Under imperfectly protected wealth, $w(t)<1$, the owner and the trespasser choose their extraction simultaneously and non-cooperatively. Extraction decisions take into account that the flow of revenues is exposed to theft, and that both the owner and the trespasser are depleting the same stock. Using the equilibrium levels of effort in the theft contests, (4) and (5), the problems of the owner and the trespasser are respectively given by the following HJB equations

$$
\begin{gathered}
r V_{i, x y}=\max _{\left\{R_{i}\right\}}\left\{\lambda_{y}^{2} \frac{R_{i}^{1-\frac{1}{\theta}}}{1-\frac{1}{\theta}}-\frac{\partial V_{i, x y}}{\partial S}\left(R_{i}+R_{j}\right)+\pi_{x y}\left(\mathbf{E}_{x y}\left[V_{i}\right]-V_{i, x y}\right)\right\}, \\
r V_{j, x y}=\max _{\left\{R_{j}\right\}}\left\{\lambda_{y}^{2}\left(1-\omega_{x}\right) \frac{R_{j}^{1-\frac{1}{\theta}}}{1-\frac{1}{\theta}}-\frac{\partial V_{j, x y}}{\partial S}\left(R_{i}+R_{j}\right)+\pi_{x y}\left(\mathbf{E}_{x y}\left[V_{j}\right]-V_{j, x y}\right)\right\} .
\end{gathered}
$$

The FOCs of these problems respectively give $\lambda_{y}^{2} R_{i}^{-\frac{1}{\theta}}=\partial V_{i, x y} / \partial S$ and $\lambda_{y}^{2}\left(1-\omega_{x}\right) R_{j}^{-\frac{1}{\theta}}=\partial V_{j, x y} / \partial S$. Back into the value functions we obtain

$$
\left(r+\pi_{x y}\right) V_{i, x y}=\frac{\lambda_{y}^{2 \theta}}{\theta-1}\left(\frac{\partial V_{i, x y}}{\partial S}\right)^{1-\theta}-\lambda_{y}^{2 \theta}\left(1-\omega_{x}\right)^{\theta} \frac{\partial V_{j, x y}}{\partial S}\left(\frac{\partial V_{j, x y}}{\partial S}\right)^{-\theta}+\pi_{x y} \mathbf{E}_{x y}\left[V_{i}\right],
$$


and

$$
\left(r+\pi_{x y}\right) V_{j, x y}=\frac{\lambda_{y}^{2 \theta}\left(1-\omega_{x}\right)^{\theta}}{\theta-1}\left(\frac{\partial V_{j, x y}}{\partial S}\right)^{1-\theta}-\lambda_{y}^{2 \theta} \frac{\partial V_{j, x y}}{\partial S}\left(\frac{\partial V_{i, x y}}{\partial S}\right)^{-\theta}+\pi_{x y} \mathbf{E}_{x y}\left[V_{j}\right]
$$

We use $V_{i, x y}=\lambda_{y}^{2} \theta(\theta-1)^{-1} S^{1-\frac{1}{\theta}} D_{i, x y}^{-\frac{1}{\theta}}$ and $V_{j, x y}=\lambda_{y}^{2}\left(1-\omega_{x}\right) \theta(\theta-1)^{-1} S^{1-\frac{1}{\theta}} D_{j, x y}^{-\frac{1}{\theta}}$ as guesses for each of the two value functions, with $D_{m, x y} \equiv R_{m, x y} / s$ denoting depletion by agent $m$ in regime $x y$. Using this we can rewrite the system of value functions as a system of reaction functions, which implicitly solves for the equilibrium depletion rates by the owner and the trespasser, $D_{i, x y}$ and $D_{j, x y}$. This system of reaction functions is given

$$
D_{i, x y}=D^{*}+(\theta-1) D_{j, x y}-\theta g_{i, x y}
$$

for the owner, and

$$
\begin{gathered}
D_{j, x y}=D^{*}+(\theta-1) D_{i, x y}-\theta g_{j, x y} \text { if } \omega_{x}<1, \\
D_{j, x y}=0 \text { if } \omega_{x}=1
\end{gathered}
$$

for the trespasser. The last line corresponds to the case with perfect wealth protection under which the trespasser lacks incentives to engage in extraction.

The terms $g_{i, x y}$ and $g_{j, x y}$ in (6) and (9) determine whether, through the lenses of the current regime $x y$, the prospect of a regime shift is seen as good $(g>0)$ or bad $(g<0)$ news, by the owner and the trespasser respectively. These terms are given by

$$
g_{i, x y} \equiv \sum_{\tilde{x} \tilde{y} \neq x y} \pi_{x y \rightarrow \tilde{x} \tilde{y}}\left(\frac{V_{i, \tilde{y} \tilde{y}}}{V_{i, x y}}-1\right)=\sum_{\tilde{x} \tilde{y} \neq x y} \pi_{x y \rightarrow \tilde{x} \tilde{y}}\left[\frac{\lambda_{\tilde{y}}^{2}}{\lambda_{y}^{2}}\left(\frac{D_{i, x y}}{D_{i, \tilde{x} \tilde{y}}}\right)^{\frac{1}{\theta}}-1\right],
$$

and

$$
g_{j, x y} \equiv \sum_{\tilde{x} \tilde{y} \neq x y} \pi_{x y \rightarrow \tilde{x} \tilde{y}}\left(\frac{V_{j, \tilde{x} \tilde{y}}}{V_{j, x y}}-1\right) ; \frac{V_{j, \tilde{x} \tilde{y}}}{V_{j, x y}}=\left\{\begin{array}{c}
\frac{\left(1-\omega_{\tilde{x}}\right) \lambda_{\tilde{y}}^{2}}{\left(1-\omega_{x}\right) \lambda_{y}^{2}}\left(\frac{D_{j, x y}}{D_{j, \tilde{y}}}\right)^{\frac{1}{\theta}} \text { if } \omega_{\tilde{x}}<1 \\
0 \text { otherwise }
\end{array}\right.
$$

where $\pi_{x y \rightarrow \tilde{x} \tilde{y}}$ is the flow probability of a shift from regime $x y$ to regime $\tilde{x} \tilde{y}$, e.g., $\pi_{00 \rightarrow 10}=\pi p(1-q)$.

Agents face uncertainty about the subsequent regime; even if they know exactly what regime comes next (e.g., regime 11 when the current regime is 10) they do not know when exactly this regime shift will occur. However, agents know whether they will be better or worse off immediately after a given regime arrives, and how likely is the arrival of every possible subsequent regime. The former is given by the rate of change in the NPV of revenues upon the arrival of regime $\tilde{x} \tilde{y}, V_{i, \tilde{x}} / V_{i, x y}-1$, and the latter by the flow probability $\pi_{x y \rightarrow \tilde{x} \tilde{y}}$. The overall expectation of the future is given by the sum of the rates of change resulting from all the possible regime shifts weighed by the likelihood of each shift. Agent $m$ considers the prospect of a regime shift as good news, $g_{m, x y}>0$, if in the current regime $x y$ the value of the remaining stock is lower than its average value in all the possible subsequent regimes. Using (8) and (9) in the system of response functions in (6) and (7) one obtains the implicit solution for the owner's and trespasser's depletion rates in equilibrium in regime $x y$. Note that in case of static institutions, or if the economy is in the absorptive regime $11, g_{i}=g_{j}=0$. 
Lemma 1. In each regime $x y \in I$, there exists a unique equilibrium with constant $D_{i, x y} \geq 0$ and $D_{j, x y} \geq 0$.

Proof. See Appendix A.2.

\subsection{Institutional dynamics}

The response functions (6) and (7) only feature the current levels of institutional strength, $\omega_{x}$ and $\lambda_{y}$, in the 'news' expressions (8) and (9). Thus, what is relevant for depletion is how the current levels of protection compare to future levels $\left(\left(1-\omega_{\tilde{x}}\right) /\left(1-\omega_{x}\right), \lambda_{\tilde{y}} / \lambda_{y}\right)$. In other words, institutional dynamics play a central role in determining the pace at which the resource is exhausted, and thus in determining how efficiently it is managed over time. To illustrate this point, in what follows characterize the effect of institutional dynamics on total depletion compared to the owner's optimal level of depletion $D^{*}$ (i.e., the level that would arise in case of perfect protection). As it turns out, we find that, under imperfect protection, institutional dynamics are a necessary condition for total depletion $D_{x y}$ not to exceed the perfect protection benchmark $D^{*}$ :

Proposition 1. In any regime $x y$ with imperfectly protected wealth, $W(t)=\omega_{x}<1$,

- if institutions are static $\left(g_{i, x y}=g_{j, x y}=0\right)$, total depletion is equal to $2 D^{*} /(2-\theta)$, and thus exceeds $D^{*}$;

- if institutions are bound to change and the prospect of an institutional regime shift is neutral or bad news either for the owner or the trespasser (i.e., $g_{i, x y} \leq 0$ or $g_{j, x y} \leq 0$ ), total depletion exceeds $D^{*}$;

- if institutions are bound to change and the prospect of an institutional regime shift is good news for both the owner and the trespasser (i.e., $g_{i, x y}>0$ and $g_{j, x y}>0$ ), total depletion will be lower than $2 D^{*} /(2-\theta)$, and may be lower than $D^{*}$.

Proof. See Appendix A.1

From this proposition it follows that not only institutional dynamics are a necessary condition for total depletion to be equal to the perfect protection benchmark, but also that the expected regime shift must be good news for both the owner and the trespasser. This last requirement, follows from the second part of the proposition and can be seen using (6) and (7); these two equations, show that if either $g_{m, x y} \leq 0$ for either $m=i$ or $m=j$, individual depletion by $m$ is not less that $D^{*}$, and hence total depletion necessarily exceeds $D^{*}$ if wealth is imperfectly protected. As to the necessity of institutional dynamics under imperfect wealth protection, in the absence of dynamics total depletion is excessive due to the common pool externality. Total depletion in this case is $2 D^{*} /(2-\theta)$, which solely reflects the distortion caused by the common pool externality.

Our take away from this proposition is the following: with institutional dynamics the imperfect protection of wealth need not result in excessive depletion. Total depletion is also affected by how agents react to the prospect of institutional regime shifts (and how they react to the other's reaction). Agents find it individually optimal to extract less intensively in regimes where the resource has a relatively low net return, and save the resource for regimes where the stock has a relatively high one. When agents interpret institutional change as good news they effectively anticipate that in the 
'average' future regime the resource is more valuable. In this case, they adopt a more conservative use of the resource in the current regime, and shift extraction towards future regimes. In other words, when the prospect of a regime shift is interpreted as good news, agents effectively become more patient and slow down current depletion. The opposite is true when they internalize the prospect of future regime shifts as bad news.

The above means that the effect of good news runs opposite to the common pool externality. According to Proposition 11 the excessive depletion induced by the externality is partially offset if both the owner and the trespasser anticipate good news $g_{i, x y}>0$ and $g_{j, x y}>0$. By adding up (6) and (7) we obtain that total depletion is

$$
D_{x y}=\frac{2 D^{*}-\theta\left(g_{i, x y}+g_{j, x y}\right)}{2-\theta} .
$$

This expression by itself highlights the role of dynamic institutions in countering the effect of the common pool externality. With $g_{i, x y}>0$ and $g_{j, x y}>0$ we obtain that total depletion is below the common pool level of depletion: $D_{x y}<2 D^{*} /(2-\theta)$. When news are sufficiently good, the good news effect may even (more than) fully offset the common pool externality: $D_{x y}<D^{*}$.

In contrast, if the current regime features perfect wealth protection, $W(t)=1$, and consequently the common pool externality is absent, dynamic institutions are no longer a necessary condition to attain the optimal level of depletion that arises under perfect protection.

Corollary 1. If institutions are static, or equivalently, the prospect of a regime shift is neutral news for the owner (i.e., $g_{i, x y}=0$ ), then total depletion coincides with the prefect protection benchmark $D^{*}$ if and only if wealth is perfectly protected $\left(w(t)=\omega_{x}=1\right)$.

Proof. Follows directly from using $D_{j, x y}=0$ and $g_{i, x y}=0$ in (6).

Proposition 1 and Corollary 1 establish the relevance of institutional dynamics to determine whether total depletion is bound to be sub-optimally high in the presence of weak institutions. The excessive depletion caused by the weak protection of wealth (trespassing), is only countered if institutions are dynamic and agents expect future institutions to be more favorable, and hence have incentives to preserve the resource for future regimes. This implies that the mere presence of imperfectly protected income, i.e., theft, does not act as a countering force to the common pool externality. Instead, to fully offset the common pool externality, income needs to be imperfectly protected and the level of protection needs to be expected to improve in the future. As established in Proposition 1, the common pool externality can only be fully offset if the prospect of a regime shift is good news for both agents. Dynamics along the wealth dimension cannot play this role, as they trigger asymmetric effects. What constitutes good news for one agent is bad for the other: the owner benefits from stronger wealth protection, but the trespasser is harmed by this. Hence, institutional dynamics can only be good news for both agents if the dynamics involve stronger income protection in future regimes. And this, by definition, requires imperfect income protection in the current regime.

With this we complete the characterization of a general regime $x y$, and can proceed to find the equilibrium of the model. We do this by solving the four institutional regimes through which the 
economy may transition. To this end we proceed backwards from the final regime 11 to the initial regime 00, going through the intermediate regimes 10 and 01 . This approach allows us to isolate the effect of institutional dynamics along the income and wealth dimensions, and then explore how dynamics along both dimensions jointly determine the level of total depletion.

\subsubsection{Static institutions (regime 11)}

In this regime, $w(t)$ and $l(t)$ have both reached their final values $\omega_{1}$ and $\lambda_{1}$, and thus regime 11 features static institutions. Proposition 1 and Corollary 1 entail that if in this final regime there is perfect protection of wealth (i.e., $\omega_{1}=1$ ) and hence no trespassing, total depletion coincides with the perfect protection benchmark, $D^{*}$; otherwise, total depletion in the final regime is sub-optimally high, due to the common pool externality. Formally,

$$
\begin{gathered}
D_{i, 11}=D_{j, 11}=\frac{D^{*}}{2-\theta} \text { if } \omega_{1}<1 \\
D_{i, 11}=D^{*}, D_{j, 11}=0 \text { if } \omega_{1}=1
\end{gathered} .
$$

Naturally, regime 11 is preceded by a regime in which institutions are bound to change. To study the effect of dynamics in each institutional dimension in isolation, we first study the case in which only income protection is dynamic (regime 10) and then the case in which dynamics are only possible along the wealth dimension (regime 01).

\subsubsection{Extraction under unstable income protection (regime 10)}

In regime 10 institutions are bound to change in the income dimension, $w(t)=\omega_{1}$ and $l(t)=\lambda_{0}$, and agents know that upon an institutional change the economy surely shifts to regime 11. In case of imperfect wealth protection, $\omega_{1}<1$, the good news expressions in equations (8) and (9) simplify to

$$
g_{m, 10}=\pi q\left[\Lambda^{2}\left(\frac{D_{m, 10}}{D_{m, 11}}\right)^{\frac{1}{\theta}}-1\right]
$$

where

$$
\Lambda \equiv \frac{\lambda_{1}}{\lambda_{0}} \geq 0
$$

is the anticipated improvement $(\Lambda>1)$ or deterioration $(\Lambda<1)$ of income protection upon an institutional change in this dimension. Under perfect wealth protection, $\omega_{1}=1$, extraction by the trespasser is zero in the current and in the subsequent regime $D_{j, 10}=D_{j, 11}=0$, and expression (12) is only relevant to determine current depletion by the owner. Using (12) in (6) and (7) we obtain

$$
D_{i, 10}=\left\{\begin{array}{c}
D^{*}+(\theta-1) D_{j, 10}-\theta \pi q\left[\Lambda^{2}\left(\frac{D_{i, 10}}{D_{i, 11}}\right)^{\frac{1}{\theta}}-1\right] \text { if } \omega_{1}<1 \\
D^{*}-\theta \pi q\left[\Lambda^{2}\left(\frac{D_{i, 10}}{D^{*}}\right)^{\frac{1}{\theta}}-1\right] \text { otherwise }
\end{array},\right.
$$


and

$$
D_{j, 10}=\left\{\begin{array}{c}
D^{*}+(\theta-1) D_{i, 10}-\theta \pi q\left[\Lambda^{2}\left(\frac{D_{j, 10}}{D_{j, 11}}\right)^{\frac{1}{\theta}}-1\right] \text { if } \omega_{1}<1 \\
0 \text { otherwise }
\end{array}\right.
$$

where and $D_{i, 11}$ and $D_{j, 11}$ are given in (11). Equation $(12)$ immediately reveals that the level of good news depends on $\Lambda$. If income protection improves (deteriorates), $\Lambda>1(\Lambda<1)$, the share of revenues effectively accrued by the owner, net of theft and protective effort, will increase (decrease) when regime 11 arrives. The same applies for the trespasser if she is active $\left(\omega_{1}<1\right)$. Thus, $\Lambda>1$ means that the prospect of institutional change from regime 10 to 11 is good news for the owner and the trespasser; according to Proposition 1 this is a necessary condition to offset the excessive depletion caused by the common pool externality. However, we have yet to have establish how good good news must be to fully offset the effect of trespassing.

Proposition 2. In regime 10, where institutions are dynamic only along the income dimension: (i) total depletion is strictly decreasing in the anticipated change in income protection $\Lambda$, for any $\Lambda>0$; and, (ii) there exists a unique threshold $\Lambda_{10}^{*}$ such that

- if $\Lambda>\Lambda_{10}^{*}$, then $D_{10}<D^{*}$,

- if $\Lambda<\Lambda_{10}^{*}$ then $D_{10}>D^{*}$, and

- if $\Lambda=\Lambda_{10}^{*}$ then $D_{10}=D^{*}$.

Where $\Lambda_{10}^{*}=\left[\left(1+D^{*}(2 \pi q)^{-1}\right) 2^{\frac{1}{\theta}}(2-\theta)^{-\frac{1}{\theta}}\right]^{\frac{1}{2}}>1$ if wealth is weakly protected; and, $\Lambda_{10}^{*}=1$ if wealth is perfectly protected.

\section{Proof. See Appendix A.1}

The improvement in income protection $(\Lambda>1)$ implies that both the owner and the trespasser perceive a larger return from extraction in the subsequent regime. This creates incentives for both agents to slow down extraction in the current regime, and these incentives are stronger the higher the value of $\Lambda$. These individual incentives are further reinforced by the strategic complementarity implied by (14) and (15), whereby a more conservative use by one agent induces more conservative use by the other. Hence a higher $\Lambda$ is associated to slower depletion in regime 10. If the improvement in income protection is sufficiently large, $\Lambda>\Lambda_{10}^{*}$, the preservation induced by the anticipation of the regime shift more than offsets the excessive depletion caused by the common pool externality caused by the weak protection of wealth.

\subsubsection{Extraction under unstable wealth protection (regime 01)}

In regime 01 the level of wealth protection is bound to change: $w(t)=\omega_{0}$ and $l(t)=\lambda_{1}$. As in regime 10, in regime 01 agents know that the current regime can only be followed by regime 11 . If wealth is imperfectly protected in regime $01, \omega_{0}<1$, the good news expressions in equations $(8)$ and (9) simplify to

$$
g_{i, 01}=\pi p\left[\left(\frac{D_{i, 01}}{D_{i, 11}}\right)^{\frac{1}{\theta}}-1\right]
$$


and

$$
g_{j, 01}=\left\{\begin{array}{c}
\pi p\left[\Omega^{-1}\left(\frac{D_{j, 01}}{D_{j, 11}}\right)^{\frac{1}{\theta}}-1\right] \text { if } \omega_{1}<1 . \\
-\pi p \text { otherwise }
\end{array} .\right.
$$

Where

$$
\Omega \equiv \frac{1-\omega_{0}}{1-\omega_{1}} \geq 0
$$

is the improvement $(\Omega>1)$ or deterioration $(\Omega<1)$ of wealth protection when institutions change in the wealth dimension. If wealth is perfectly protected in regime 01 , extraction by the trespasser is zero, and only equation (16) is relevant. Using (16) and (17) in (6) and (7) we obtain

$$
D_{i, 01}=D^{*}+(\theta-1) D_{j, 01}-\theta \pi p\left[\left(\frac{D_{i, 01}}{D_{i, 11}}\right)^{\frac{1}{\theta}}-1\right] \text {, }
$$

and

$$
D_{j, 01}=\left\{\begin{array}{c}
D^{*}+(\theta-1) D_{i, 01}-\theta \pi p\left[\Omega^{-1}\left(\frac{D_{j, 01}}{D_{j, 11}}\right)^{\frac{1}{\theta}}-1\right] \text { if } \omega_{0}<1 \text { and } \omega_{1}<1 \\
D^{*}+(\theta-1) D_{i, 01}+\theta \pi p \text { if } \omega_{1}=1 \\
0 \text { if } \omega_{0}=1
\end{array} ;\right.
$$

with $D_{i, 11}$ and $D_{j, 11}$ given by (11). From equations (16)-(19) one can see that institutional dynamics along the wealth dimension have a direct impact on the trespasser's extraction decision. If wealth protection is expected to improve, $\Omega>1$, the trespasser's capacity to accrue revenues from resource extraction deteriorates upon the regime shift from 01 to 11 . Hence the trespasser perceives the prospect of institutional change as bad news, and engages in a less conservative use of the resource in the current regime. The opposite is true in case of an anticipated deterioration, $\Omega<1$. From the perspective of the owner, the evolution of wealth protection does not have a direct incidence on the extraction decision. Nonetheless, extraction by the owner is indirectly affected through the strategic interaction with the trespasser. For a given $D_{i, 01}$, a higher $\Omega$ results in a higher depletion by the trespasser $D_{j, 01}$. This is reinforced by the strategic complementarity implied by (18): higher depletion by the trespasser is met with higher depletion by the owner. As a result, in equilibrium, the prospect of an improvement in wealth protection, $\Omega>1$, results in a less conservative use of the resource by both the owner and the trespasser in regime 01 , relative to what would occur under static institutions $(\Omega=1)$. The opposite is true in case of a deterioration of wealth protection between regimes 01 and 11: $\Omega<1$. Following this, the role of $\Omega$ is opposite to that of $\Lambda$, as formalized in the following proposition.

Proposition 3. In regime 10, where institutions are dynamic only along the wealth dimension: (i) total depletion is strictly increasing in the anticipated change in wealth protection $\Omega$, for any $\Omega \in(0, \infty)$; and, (ii) total depletion exceeds the perfect protection benchmark, $D_{01}>D^{*}$.

Proof. See Appendix A.1

Proposition 1 establishes that institutional dynamics are necessary to offset the excessive depletion caused by the common pool externality, and the prospect of institutional change, brought about 
by these dynamics, must constitute good news for both the owner and the trespasser. The latter condition cannot be met in regime 01 . If wealth is imperfectly protected in this regime, the prospect of institutional change that constitutes good news for one agent simultaneously reads as bad news to the other: for instance, $\omega_{1}>\omega_{0}$ is good news for the owner and bad news for the trespasser. Interestingly, institutional dynamics imply that if wealth is perfectly protected in this regime, $\omega_{0}=1$, depletion will exceed $D^{*}$. With $\omega_{0}=1$, dynamics imply an eventual shift towards a regime with $\omega_{1}<1$; the owner internalizes this as bad news, $g_{i, 01}<0$ : currently there is no trespassing but in the subsequent regime there will be. Despite the current absence of the trespasser, the shadow of its presence in the future, pushes the owner towards excessive depletion in regime 01 . Thus, in regime 01 total depletion is excessive irrespective of the current level of wealth protection $\omega_{0}$.

This completes the characterization of the effect of institutional dynamics on depletion in the intermediate regimes 10 and 01 , where there are dynamics only along one of the two institutional dimensions. Now we can move backwards, to the initial institutional regime, 00, where institutions are bound to evolve along both the wealth and income dimension.

\subsubsection{Extraction under unstable wealth and income protection (regime 00)}

In the initial regime, 00, wealth and income protection are at their initial states: $w(t)=\omega_{0}$ and $l(t)=\lambda_{0}$. As opposed to regimes 10 and 01 , in this regime agents do not know with exactly what will be the subsequent regime. However, agents anticipate that income and wealth protection will evolve, as captured by $\Lambda \neq 1$ and $\Omega \neq 1$. From the view point of regime 00 , the anticipated changes in income and wealth protection $\Lambda$ and $\Omega$ play a similar role as they respectively do in regimes 10 and 01. A higher anticipated change in income protection, $\Lambda$, makes the prospect of institutional change better news to both agents, which mitigates the common pool externality.

Proposition 4. In the initial regime 00, where institutions are dynamic along both dimensions: (i) total depletion is (strictly) decreasing in the anticipated change in income protection, $\Lambda$, for any $\Lambda \geq 0$ ( $\Lambda>0$ ); and, (ii) for any given anticipated improvement/deterioration in wealth protection, $\Omega$, there exists a unique threshold $\Lambda_{00}^{*}(\Omega)>1$ such that

- if $\Lambda>\Lambda_{00}^{*}(\Omega)$, then $D_{00}<D^{*}$,

- if $\Lambda<\Lambda_{00}^{*}(\Omega)$, then $D_{00}>D^{*}$, and

- if $\Lambda=\Lambda_{00}^{*}(\Omega)$, then $D_{00}=D^{*}$.

Proof. See Appendix A.1

This proposition generalizes the results in Proposition 2, for any anticipated change in wealth protection, and highlights the importance of institutional dynamics to offset the effect of the common pool externality. Again, the prospect of institutional change, not only needs to be anticipated as good news by both the owner and the trespasser, but also it needs to be sufficiently good news: $\Lambda>\Lambda_{00}^{*}>1$.

In terms of wealth protection, a higher $\Omega$ implies that the prospect of institutional change is worse news for the trespasser, which consequently engages in more rapacious extraction in the current regime. Through the strategic complementarity this is met by more rapacious depletion by the owner. Thus, a higher $\Omega$ results in a less conservative use of the resource. Formally. 
Proposition 5. In the initial regime 00, where institutions are dynamic along both dimensions: (i) total depletion is strictly increasing in $\Omega=\frac{1-\omega_{0}}{1-\omega_{1}}$, for any $\Omega \in(0, \infty)$.

Proof. See Appendix A.1

In combination, Propositions 2, 4, and 5 imply that

Proposition 6. (i) The $\Lambda_{00}^{*}(\Omega)$ threshold is strictly increasing in the anticipated change in wealth protection, $\Omega$; and, (ii)

- if $\Omega>1$, then $\Lambda_{00}^{*}(\Omega)>\Lambda_{10}^{*}$,

- if $\Omega \in(0,1)$, then $\Lambda_{00}^{*}(\Omega)<\Lambda_{10}^{*}$, and

- if $\Omega=1$, then $\Lambda_{00}^{*}(\Omega)=\Lambda_{10}^{*}$.

Proof. The first part of the proposition follows from $d D_{00} / d \Lambda<0 \forall \Lambda>0$ and $d D_{00} / d \Omega>0$. For the second part note that with $\Omega=1$ regime 00 collapses into regime 10 , hence $\Lambda_{00}^{*}(1)=\Lambda_{10}^{*}$. Given that $\Lambda_{10}^{*}$ is independent of $\Omega$ and that there is a positive relationship between $\Lambda_{00}^{*}$ and $\Omega$, then it must be that $\Lambda_{00}^{*}(\Omega) \gtrless \Lambda_{10}^{*}$ if $\Omega \gtrless 1$.

Proposition 6 illustrates the interaction between the two institutional dimensions, and how their evolution can mitigate or exacerbate the common pool externality. The higher the anticipated improvement of wealth protection (i.e., the higher $\Omega$ ), the higher the return of extraction for the trespasser in the initial regime, relative to future regimes; this exacerbates the common pool externality in the initial regime. Conversely, the higher the anticipated improvement of income protection, the lower the return of extraction for both the owner and the trespasser in the initial regime, relative to future regimes; this mitigates the effect of the common pool externality in the initial regime. Take together, this implies that the higher the anticipated improvement in wealth protection $\Omega$, the higher the anticipated improvement in income protection $\Lambda$ required to offset the common pool externality.

From Propositions 2 and 4 we know that an anticipated improvement in income protection is necessary to offset the current, or future, common pool externality: $\Lambda_{00}^{*}>1$, even if $\omega_{0}=1$, and $\Lambda_{10}^{*}>1$ if $\omega_{1}<1$. Proposition 6 establishes that the income protection improvement required to offset the common pool externality needs to be higher if institutions are also expected to improve along the wealth dimension: $\Lambda_{00}^{*}(\Omega)>\Lambda_{10}^{*}$ if $\Omega>1$. Overall, this means that when it comes to determining the total level of depletion in regime 00 , institutional dynamics in one of the dimensions offsets rather that exacerbate the effects of dynamics in the other. A given level of total depletion in regime 00 will emerge under different combinations of anticipated wealth and income protection. Such combinations feature higher levels of anticipated income protection improvement $\Lambda$, the higher the anticipated improvement in wealth protection $\Omega$.

\section{Numerical example and discussion}

In this section we perform a numerical analysis of the model to gain further understanding about the relevance of institutional dynamics. This analysis consists of simulating full transitions from regime 
00 to regime 11. We set $\theta=1.5, r=5 \%, \pi=0.1, p=0.7$ and $q=0.5$. With these parameters the yearly depletion rate under perfect protection is $7.5 \%$, the expected duration of $w(t)=\omega_{0}$ is 14.3 years, and of $l(t)=\lambda_{0}$ is 20 years. We discuss two scenarios, one of general institutional deterioration with $\omega_{0}=\lambda_{0}=1$ and $\omega_{1}=\lambda_{1}=0.8$, and one of general improvement with $\omega_{0}=\lambda_{0}=0.8$ and $\omega_{1}=\lambda_{1}=1$. The initial stock, $S_{0}$ is set to 100 .

We compare the 'dynamic institutions' paths (represented in blue) with two 'static institutions' paths: optimal and common pool. The optimal path is the best possible path for the owner. It corresponds to the perfect protection benchmark described above, where wealth and income are permanently perfectly protected (i.e., $w(t)=l(t)=1$ for all $t$ ) and thus, there is neither trespassing nor theft (green dashed curves). The common pool path illustrates a scenario with perfect income protection and imperfect wealth protection, where the imperfect protection of wealth remains equally intense over time (i.e., $w(t)=\bar{\omega}<1$ and $l(t)=1$ for all $t$ ); in this case, the management of the resource is only distorted by the common pool externality (red dot-dashed curves). In the simulations presented below, we assume that institutions follow the expected path; that is, the economy shifts from regime 00 to regime 10 in year 14, and from regime 10 to regime 11 in year 20. Based on our definition of the benchmark with perfect institutions the analysis revolves around the equilibrium outcomes for the owner.

Depletion Our analytical results show the effect of dynamic institutions on total depletion, and how the rate of depletion changes in the different regimes through which the economy may transition. In this numerical exercise, we further illustrate the impact of institutional dynamics, on the evolution of the remaining stock in the ground (Figure 1) and the total depletion rate (Figure 2). We compare these to the evolution of the stock under the optimal path, where total depletion is equal to $D^{*}$, and under the common pool path, where total depletion is $2 D^{*} /(2-\theta)$.

With deteriorating institutions, in the initial regime 00 we observe a fast decline in the stock of the resource, relative to the optimal path (Figure 19). This is in line with Proposition 4 which establishes that the anticipated weakening of income protection implies total depletion in excess of $D^{*}$ in regime 00. Despite the perfect protection in the initial regime, the anticipation of an institutional deterioration, which reads as bad news, pushes the owner to engage in excessive depletion (Figure 22). Nevertheless, the level of bad news embedded in the anticipated weakening of institutions is not sufficient to push depletion above the common pool level of depletion. Total depletion in regime 00 is about two times the depletion in the optimal path and about half of the depletion in the common pool path (Figure 2 2 ). With the arrival of the intermediate regime 10 the trespasser becomes active. From the perspective of this regime, the shift to regime 11 would entail a weakening of income protection, and hence the prospect of the eventual shift to regime 11 is bad news for both the owner and the trespasser. In combination, the weak protection of wealth and the prospect of a weaker protection of income, accelerate the resource stock decline with respect to the initial regime (Figure 13 ), and total depletion exceeds the common pool level (Figure 2a). This is in line with the result in (10). When regime 11 arrives income and wealth protection are in their final state, and institutions become static. As stated in Proposition 1, the absence of institutional dynamics and the imperfect protection of wealth, imply that total depletion in this last regime corresponds to the common pool level. 
Figure 1: Remaining stock $(S)$

(a) Deterioration

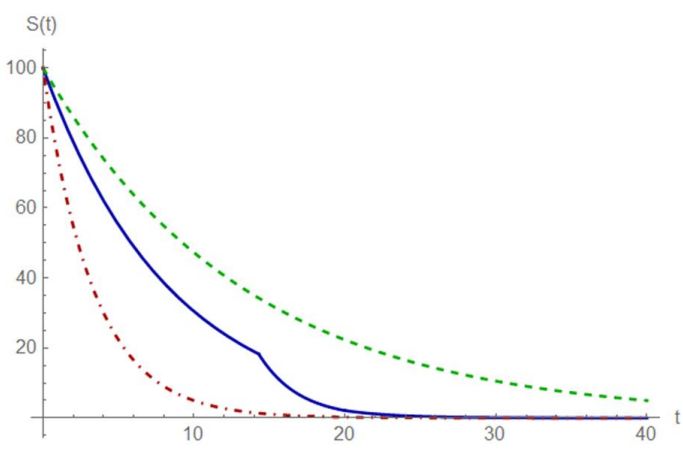

(b) Improvement

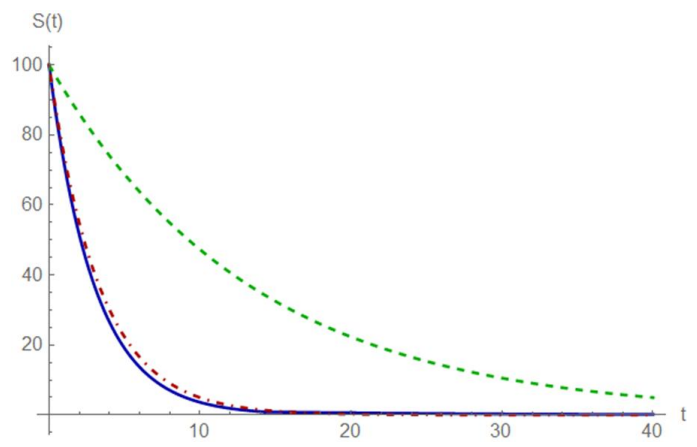

Figure 2: Total depletion relative to optimum $\left(D_{x y} / D^{*}\right)$

(a) Deterioration

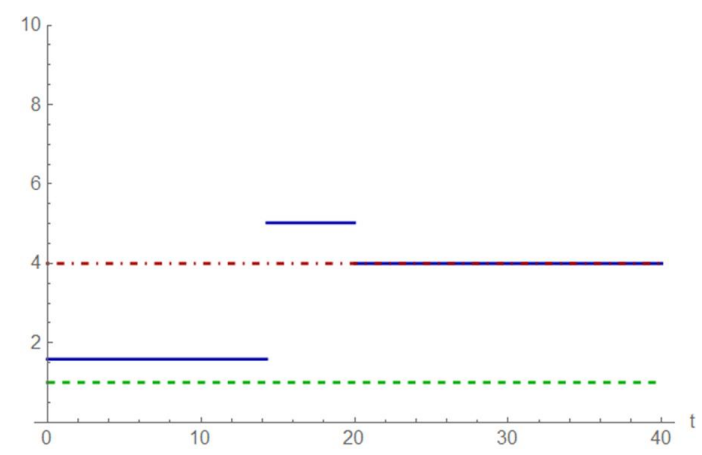

(b) Improvement

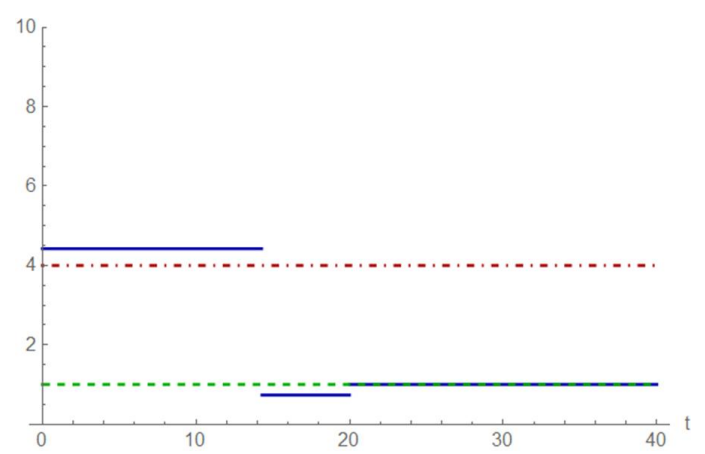

-...- Optimal

..... Common Pool

_ Dynamic Institutions 
In case of improving institutions, the initial regime features a quick decline of the resource stock (Figure 1p), with total depletion even exceeding depletion under the common pool path (Figure 2p). This is the result of the less conservative use by the trespasser in anticipation of the strengthening of wealth protection, which turns out to be the dominant force in determining total depletion in the initial regime. Under our parameterization a regime shift along the wealth dimension amounts to leaving the trespasser without access to the resource, which constitutes 'very bad' news for this agent. These news dominate over the good news embedded in the anticipated strengthening of income protection; the end result is a rapacious use of the resource by the trespasser. In terms of total depletion, this is reinforced by the strategic complementarity between the owner's and trespasser's extraction, and partially mitigated by the owner's higher effective patience induced by the anticipation of the institutional strengthening along the two dimensions. Upon the arrival of regime 10 wealth becomes perfectly protected and only the owner is active. In this regime the owner's extraction is distorted by the anticipation of stronger income protection in the future which results in a conservative use of the resource, so that proportionally more is extracted under stronger income protection. As a consequence, in regime 10 extraction falls below the optimal level (Figure $2 \mathrm{p}$ ). Finally, with the arrival of regime 11 institutions become static and given that wealth is perfectly protected depletion coincides with the optimal level as established in (11). This, however, does not mean that the owner is equally well off than under the optimal path: the transition towards regime 11 leaves as a legacy a largely depleted resource stock (Figure $1 \mathrm{~b}$ ).

Cost of institutional instability In regimes with weak institutions the owner cannot fully accrue the potential benefits of owning the resource stock: a fraction of the stock is extracted by the trespasser and part of the revenues from extraction are captured by the thief. In addition to this, the anticipated evolution of institutions distorts the intertemporal trade-off faced by the owner when deciding current and future extraction, and this limits the owner's capacity to fully benefit from exploiting the resource even if it is currently perfectly protected. Relative to the optimal path, these distortions naturally result in a lower expected value of the remaining stock for the owner. This is irrespective of whether institutions follow a deterioration or improvement path as shown in Figure 3 . The expected value of the remaining stock can be interpreted as the maximum price that a risk-averse agent will be willing to pay to own the exploitation rights of the remaining resource stock, given the current and anticipated protection of rights over the stock and the revenues from extracting it. This illustrates how challenging it can be to obtain benefits out of exploiting or licensing the exploitation rights of nonrenewable resources in settings with institutional instability, where either institutions are currently weak or bound to weaken. Under a institutional deterioration, Figure 3 , the expected effect of weaker institutions is visible at $t=0$ where wealth and income are perfectly protected, but the expected value of the stock is about $75 \%$ of the value under the optimal path. This loss of value of the remaining stock is further reinforced by the relatively fast depletion of the resource within regimes and the regime shifts which result in an immediate reduction of the value of the remaining stock. Under an institutional improvement, Figure 3 , the excessively high extraction by the trespasser in in regime 00 causes the expected value of the resource for the owner to be about $50 \%$ of the value under 
Figure 3: Value of the remaining stock $\left(V_{i}(S)\right)$

(a) Deterioration

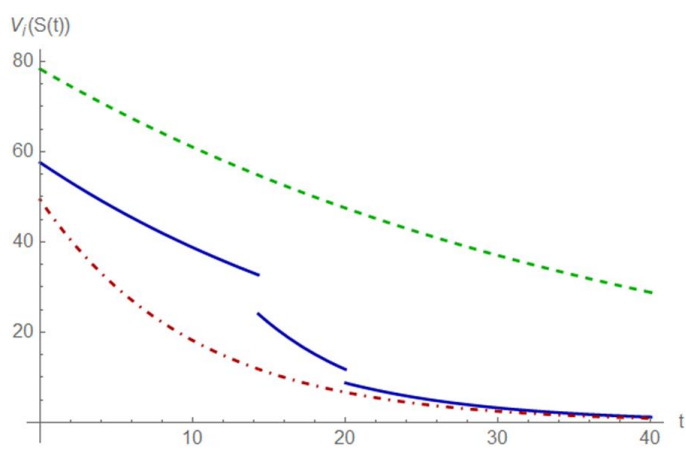

(b) Improvement

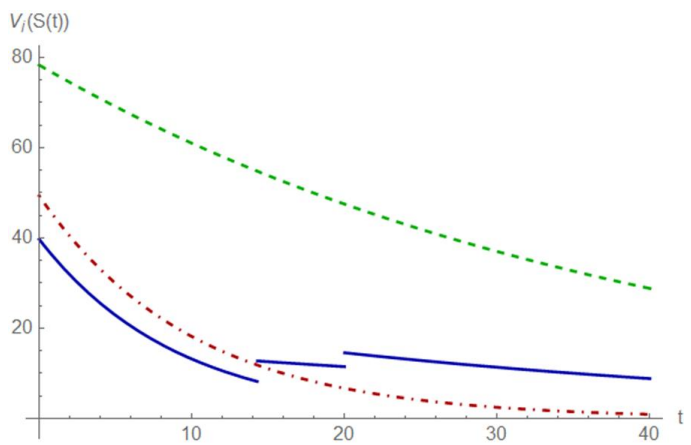

-...- Optimal

_- Dynamic Institutions

Figure 4: Stock loss equivalent $(\Delta)$

(a) Deterioration

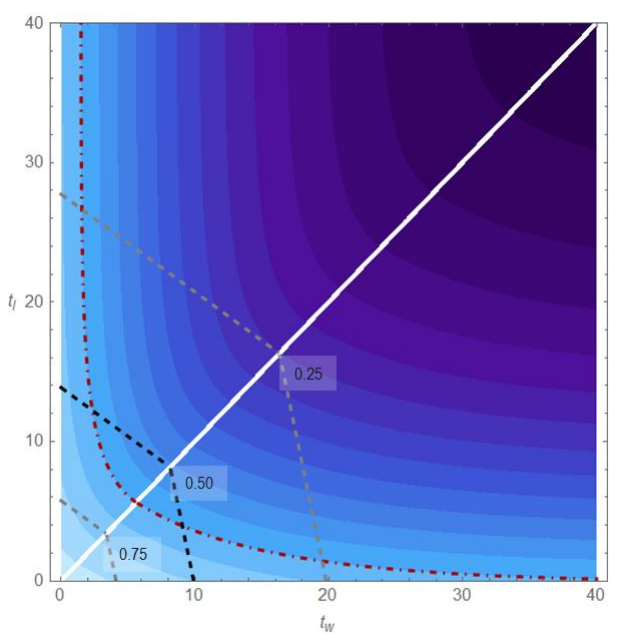

(b) Improvement

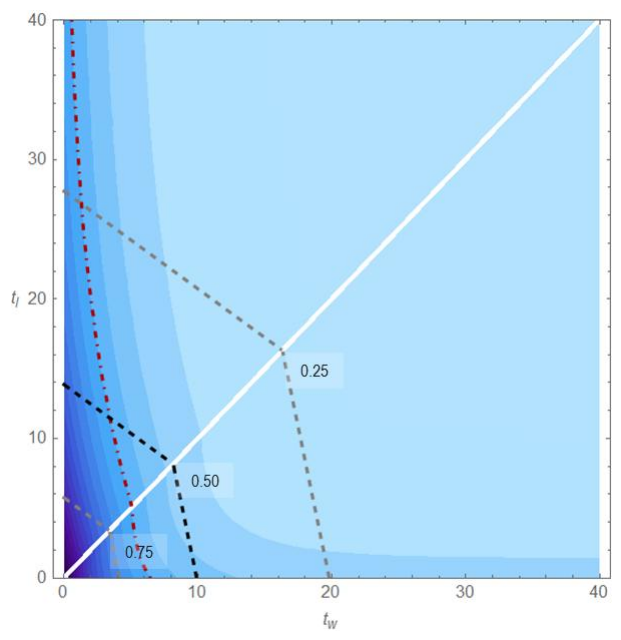

Common Pool

Survival $50 \%$

Survival $25 / 75 \%$ 
the optimal path at time $t=0$, which is even lower than the value under the common pool path. This loss in value of the remaining stock under dynamic institutions gets accentuated within regime 00 as the resource is quickly depleted. As opposed to the institutional deterioration, in case of improvement, the regime shifts manifest as a discrete increase in the value of the resource. These shifts, and the relatively conservative use in regime 10 serve to partially close the gap with respect to the optimal path.

The results in Figure 3 correspond to the evolution of the expected value (i.e., the ex-ante value) of the resource stock when the institutional regime shifts follow the expected timing. Evidently, this is not the only possible timing of events, and whether institutions are faster or slower to change is important in determining the effective losses that the owner will face under institutional dynamics. For instance, in case of institutional deterioration a slower transition out of regime 00 is beneficial for the owner, whereas the opposite is true in case of improvement.

To quantify the losses for the owner under different realizations of the timing of the regime shifts, we construct a stock-loss equivalent. For this, we first calculate the NPV of revenues that a given realization of the timing of the shifts $\left(t_{w}, t_{l}\right)$ delivers; where $t_{w} \geq 0$ denotes the timing of the shift in protection in the wealth dimension from $\omega_{0}$ to $\omega_{1}$, and $t_{l} \geq 0$ denotes the timing of the shift in the income dimension from $\lambda_{0}$ to $\lambda_{1}$. As in the preceding simulations, we assume that in case of a deterioration $\omega_{0}=\lambda_{0}=1$ and $\omega_{1}=\lambda_{1}=0.8$, and the opposite in case of an improvement. Then, we calculate the fraction of the remaining stock the owner should lose at $t=0$ such that under the optimal path the NPV of the revenues from the stock net of this loss coincides with the NPV of revenues delivered by the full resource stock under institutional dynamics and a given realization $\left(t_{w}, t_{l}\right)$. Formally, and denoting the former as $V\left(S_{0} \mid t_{w}, t_{l}\right)$, the stock-loss equivalent is the $\Delta\left(t_{w}, t_{l}\right)$ that solves $V\left(S_{0} \mid t_{w}, t_{l}\right)=V_{i}^{*}\left(\left(1-\Delta\left(t_{w}, t_{l}\right)\right) S_{0}\right)$. As a reference we also compute the stock-loss equivalent of the common pool path which is defined as $\Delta_{c p}: V_{i, c p}\left(S_{0}\right)=V_{i}^{*}\left(\left(1-\Delta_{c p}\right) S_{0}\right)$.

Figure 4 presents the stock loss equivalent, where the lighter the shade the higher $\Delta$. Above the diagonal we have the situations where the economy transitions through regime 10, and below those where the transitional regime is 01 . The red dot-dashed curve represents all the combinations of $t_{w}$ and $t_{l}$ that would result in the same stock-loss equivalent as the common pool path: $\Delta\left(t_{w}, t_{l}\right)=\Delta_{c p}$. In case of institutional deterioration, slower transitions are favorable to the owner as can be seen from the stock-loss equivalent declining monotonically in $t_{w}$ and $t_{l}$ (Figure $4 \mathrm{l}$ ). As seen in the figure there are paths with substantial costs for the owner where the stock-loss equivalent is greater that in the common pool path, where the loss is equivalent to $75 \%$ of the initial stock. The realizations with high losses are those where the economy departs regime 00 relatively quickly, irrespective of whether the economy transitions through 10 or 01 . A quick arrival of regime 10 implies that the trespasser becomes active, and with that the common pool externality is at play. As seen in Figure $2 a$ in this intermediate regime the common pool externality is exacerbated by the anticipated deterioration in of income protection and the strategic complementarity in extraction; thus a path with an early arrival of 10 is associated with high losses for the owner. In case of an early shift from regime 00 to 01 , the high losses for the owner originate in the loss of revenues to theft from early on in the transition, and the distortion induced by the anticipation of the deterioration of wealth protection.

Given the constant hazard of a regime shift in each dimension, earlier transitions are more likely 
as the probability that the economy remains in the initial state in a given dimension at least up to time $t$ is strictly declining in $t$. This is illustrated by the dashed gray/black curves depicting the joint survival function: the dashed black curve depicts all the pairs $\left(t_{w}^{\prime}, t_{l}^{\prime}\right)$ such that the probability that the economy experiences the shift in the wealth dimension after $t_{w}^{\prime}$ and in the income dimension after $t_{l}^{\prime}$ is $50 \%$. Thus, the scope there is a significant scope for quick transitions with large losses. But, even if the economy experiences an unlikely slow transition, losses can be important. Our results indicate that for a sufficiently long duration of regime 00 , the stock-loss equivalent is about $7 \%$. This loss can be fully attributed to the distortion in the extraction path caused by the anticipation of an institutional deterioration. That is, these are paths in which ex-post the transition takes long, but this is unknown by the owner ex-ante, whom instead internalizes that every instant there is a constant hazard of the materialization of the institutional deterioration.

An opposite picture emerges in case of improving institutions, Figure $4 \mathrm{p}$, where the owner benefits from relatively quick transitions. Nonetheless, the severity of the common pool externality in this setting, where the anticipation of the strengthening in the wealth dimension triggers a rapacious behavior by the trespasser, implies that if institutions are slow to change in along the wealth dimension, losses for the owner can be significant. When the change from $\omega_{0}$ to $\omega_{1}$ takes more than 6 years the stock-loss equivalent for the owner will be greater than the $75 \%$ under the common pool path, even if income becomes perfectly protected already at $t=0$. This also implies that the stock-loss equivalent increases rapidly with the duration of the transition, which causes that realizations of $\left(t_{w}, t_{l}\right)$ that can be reached with at least $25 \%$ probability can deliver losses equivalent to more than $90 \%$ of the initial stock.

These results demonstrate how challenging it can be to benefit from the endowment of nonrenewable resource riches when the institutional setting is unstable. If current institutions are strong, the threat of an institutional deterioration distorts the resource use even before the deterioration materializes. If institutions are weak, a potential institutional improvement can create conflicting interests among the resource users: a stronger wealth protection benefits the owner but is detrimental for the trespasser. In such circumstances, the anticipation of an improvement may result in an excessively fast depletion of the resource which can severely limit the possibilities to benefit from resource ownership.

\section{Concluding remarks}

Weak property rights in the management of non-renewable resources goes beyond the common access (or trespassing) problem. The history of resource rushes provides prominent examples of cases in which the legitimate owners (users) of a resource not only have to deal with the weak protection of the resource wealth in the ground but also with the risk of losing the revenues from exploiting the resource. Such has been the case in the recent gold rush in Latin America. Beyond the presence of these two manifestations of weak institutions, namely the weak protection of the wealth in the ground and of the stream of revenues from extracting it, the path that institutions follow over time, and how agents anticipate this institutional dynamics can have a significant effect on the inter-temporal trade-

offs governing the choice of an extraction path. This will ultimately limit the possibilities to benefit 
from exploiting non-renewable resource riches.

The framework that we put forward in this paper highlights that the implications of considering institutional dynamics are rich and go beyond a simple offsetting between imperfections. These implications are rooted in the dynamic strategic interactions between agents, as well as in the prospect of shifts towards regimes that may be more or less favorable. We find that in the presence of weak wealth protection the ensuing excessive depletion caused by the common pool externality can only be offset if institutions are dynamic and the prospect of institutional change is favorable to the resource users: when favorable changes are anticipated, agents effectively become more patient and engage in a more conservative use of the resource. However, not all the forms of institutional change have symmetric effects, and what would be beneficial to one agent may be detrimental to the other. Specifically, while an improvement in theft protection is favorable to both the owner and the trespasser, an improvement in wealth protection favorable to the owner and detrimental to the trespasser. This means that when it comes to determining the total level of depletion, institutional dynamics in one of the dimensions offsets rather than exacerbate the effects of dynamics in the other. A given level of total depletion can be attained when either high or low improvements along the two dimensions are anticipated.

As agents anticipate future institutional changes, the imperfect protection of wealth and income will play a role in determining the current level of depletion even if both wealth and income are perfectly protected. Starting from a regime with perfect wealth and income protection, but where institutions are anticipated to deteriorate can result in substantial losses for the resource owner. These losses already take effect before the anticipated institutional deterioration actually occurs, as a consequence of the distortion in the extraction decisions caused by the anticipated institutional deterioration. The realization of the deterioration exacerbates the losses. Overall, an institutional deterioration path, that starts from a situation of perfect protection, can result substantial losses to the owner. These losses are comparable to those that would have occurred in a situation with static institutions, and a permanent exposure to trespassing.

Interestingly, a related argument applies to a process of institutional improvement. Where even if the economy eventually reaches a situation of perfect protection, the anticipated institutional transition, in particular the anticipation of a strengthening of wealth protection can exacerbate the common pool externality while the resource is exposed to trespassing. Given the non-renewable nature of the resource, the transitional exacerbation of the common pool distortion can have significant permanent effects. These arguments illustrate some of the implications of weak institutions that may be lost when institutional dynamics are unaccounted for. They speak to the relevance of considering institutional dynamics when identifying the limits to benefit from non-renewable resource endowments in weakly institutionalized economies.

The type of dynamic framework that we develop in this paper, considering different dimensions of property rights and allowing for institutional dynamics, could be applied to other resource management settings. For instance, this type of framework can be applied to future research on the interaction between governments and private extraction companies, and how this interaction is affected by the different alternatives that governments can use to retain a share of the revenues from exploitation. From the perspective of the extraction company, expropriation can be the source of insecure 
wealth, and revenue taxation the equivalent of insecure income. The interesting feature of a setup along these lines is the dual role of the government as "trespasser" and "thief". Such a model may shed light on the tools that a government can, and should, use to maximize the net present revenues that it gets from the riches in the ground. Also the framework can serve to characterize the trade-offs involved in setting up the revenue taxation regime and the expropriation protection provisions. 


\section{References}

Banchirigah, S. M. (2008). Challenges with Eradicating Illegal Mining in Ghana: A Perspective From the Grassroots. Resources Policy, 33(1), 29-38.

Bull, B., \& Rosales, A. (2020). Into the shadows: sanctions, rentierism, and economic informalization in Venezuela. European Review of Latin American and Caribbean Studies, 109, 107-133.

Channing, M., \& Clough, C. (2017). Transnational crime and the developing world (Tech. Rep.). Global Financial Integrity.

Copeland, B. R., \& Taylor, M. S. (2009). Trade, Tragedy, and the Commons. American Economic Review, 99(3), 725-749.

Croutzet, A., \& Lasserre, P. (2017). Optimal completeness of property rights on renewable resources in the presence of market power. Resource and Energy Economics, 49, 16-32.

Fox, E. (2012, 05). Mexico mining ops pay hefty extortion fees to cartels. InSight Crime. Retrieved from https://www.insightcrime.org/news/brief/mexico-mining-ops-pay-hefty -extortion-fees-to-cartels/

Hardin, G. (1968). The Tragedy of the Commons. science, 162(3859), 1243-1248.

Hilson, G. (2002). Small-scale Mining and its Socio-economic Impact in Developing Countries. Natural Resorces Forum, 26, 3-13.

Hilson, G., \& Potter, C. (2003). Why is Illegal Gold Mining Activity so Ubiquitous in Rural Ghana? African Development Review, 15(2-3), 237-270.

Hirshleifer, J. (1989). Conflict and rent-seeking success functions: Ratio vs. difference models of relative success. Public choice, 63(2), 101-112.

Hotte, L., McFerrin, R., \& Wills, D. (2013). On the Dual Nature of Weak Property Rights. Resource and Energy Economics, 35(4), 659-678.

Idrobo, N., Mejía, D., \& Tribin, A. M. (2014). Illegal gold mining and violence in colombia. Peace Economics, Peace Science and Public Policy, 20(1), 83-111.

Lipsey, R. G., \& Lancaster, K. (1956). The General Theory of Second Best. The Review of Economic Studies, 24(1), pp. 11-32.

OHCHR. (2020). Independence of the justice system and access to justice in the Bolivarian Republic of Venezuela, including for violations of economic and social rights, and the situation of human rights in the Arco Minero del Orinoco region (A/HRC No. 44/54).

Ostrom, E. (2008). Tragedy of the Commons. The New Palgrave Dictionary of Economics, 3573-3576.

Pachico, E. (2012, 05). Mexico cartels stealing gold to launder drug money. InSight Crime. Retrieved from https://www.insightcrime.org/news/brief/mexico-cartels-stealing-gold -to-launder-drug-money/

Rosales, A. (2017). Venezuela's Deepening Logic of Extraction: The Maduro government's decision to push ahead with the Orinoco Mining Arc initiative shows that extractive capitalism is alive and well in Venezuela. NACLA Report on the Americas, 49(2), 132-135.

Rosales, A. (2019). Radical rentierism: gold mining, cryptocurrency and commodity collateralization in Venezuela. Review of International Political Economy, 26(6), 1311-1332.

Sakamoto, H. (2014). Dynamic Resource Management Under the Risk of Regime Shifts. Journal of Environmental Economics and Management, 68(1), 1-19. 
Soud, D. (2019). The gold rush in Venezuela could destabilize Latin America and the Caribbean (Payne Institute commentary serie: viewpoint). The Payne Institute for Public Policy - Colorado School of Mines.

Van Long, N. (2011). Dynamic Games in the Economics of Natural Resources: A Survey. Dynamic Games and Applications, 1(1), 115-148.

Wagner, L. (2016). Organized crime and illegally mined gold in latin america. Global Initiative against Transnational Organized Crime, Geneva.

Wilburn, D. (2014). Events Affecting Gold Exploration in Venezuela Since 1999 (Open-File Report No. 2014-1068). USGS. 


\section{Appendix}

\section{A Proofs}

\section{A.1 Propositions}

\section{Proposition 1}

Proof. For the first part of the proposition, solving for $D_{i, x y}$ and $D_{j, x y}$ in (6) and (7) with $g_{i, x y}=g_{i, x y}=$ 0 , yields $D_{i, x y}=D_{j, x y}=D^{*} / 2-\theta \rightarrow D_{x y}=2 D^{*} /(2-\theta)$. For the second part, from (6) and (7), if $g_{m, x y} \leq 0$ and given that $D_{\not h, x y} \geq 0$, it follows that $D_{m, x y} \geq D^{*}$; given that $\omega_{x}<1, D_{m, x y}>0$ implies $D_{\not h, x y}>0$, so total depletion $D_{m, x y}+D_{\not h, x y}$ exceeds $D^{*}$.

From this it is immediate that $g_{i, x y}>0$ and $g_{j, x y}>0$ are necessary for total depletion $D_{x y}$ to be below $D^{*}$. Adding (6) and (7) and solving for $D_{x y}$ we obtain that

$$
D_{x y}=\frac{2 D^{*}-\theta\left(g_{i, x y}+g_{j, x y}\right)}{2-\theta} .
$$

Hence, $D_{x y} \leq D^{*}$ requires $g_{i, x y}+g_{j, x y} \geq D^{*}$, and from the second part of the proposition it also requires $g_{i, x y}>0$ and $g_{j, x y}>0$.

\section{Proposition 2}

Proof. Part (i). With $w(t)=\omega_{1}<1$, from (11) we have that $D_{i, 11}=D_{j, 11}=D^{*} /(2-\theta)$. Using the symmetry between (14) and (15), we obtain that $D_{i, 10}=D_{i, 10}=D_{10} / 2$. Plugging this into (14) and taking the total differential with respect to $\Lambda$ and solving for $d D_{10} / d \Lambda$

$$
\frac{d D_{10}}{d \Lambda}=-\frac{2 \theta \pi q \Lambda\left(\frac{D_{10} / 2}{D^{*} /(2-\theta)}\right)^{\frac{1}{\theta}}}{2-\theta+\pi q \Lambda^{2} \frac{\left(D_{10} / 2\right)^{\frac{1}{\theta}-1}}{\left(D^{*} /(2-\theta)\right)^{\frac{1}{\theta}}}} \leq 0,
$$

for any $\theta \in(1,2)$, and which holds with strict inequality for $\Lambda>0$.

With $w(t)=\omega_{1}=1$, the trespasser is not active in regime $10, D_{j, 10}=0$ and $D_{i, 10}=D_{10}$. Using this in (14) and taking the total differential with respect to $\Lambda$, and solving for $d D_{10} / d \Lambda$

$$
\frac{d D_{10}}{d \Lambda}=\frac{-2 \theta \pi q \Lambda\left(\frac{D_{10}}{D^{*}}\right)^{\frac{1}{\theta}}}{1+\pi q \Lambda^{2} \frac{D_{10}^{\frac{1}{\theta}-1}}{D^{* \frac{1}{\theta}}}} \leq 0
$$

with strict inequality for $\Lambda>0$.

Part (ii). With $w(t)=\omega_{1}=1$, from the symmetry between (14) and 15), we obtain that $D_{10}=D^{*}$ if and only if $D_{i, 10}=D_{j, 10}=D^{*} / 2$. Using $D_{i, 10}=D_{j, 10}=D^{*} / 2$, and the solution to $D_{i, 11}$, given by expression (11), in (14) and solving for $\Lambda$ we obtain 


$$
\Lambda_{10}^{*}=\left[\left(1+\frac{D^{*}}{2 \pi q}\right)\left(\frac{2}{2-\theta}\right)^{\frac{1}{\theta}}\right]^{\frac{1}{2}}>1
$$

which together with $d D_{10} / d \Lambda<0$ for $\Lambda>0$ delivers the results of part (ii) for $w(t)=\omega_{1}<1$.

With $w(t)=\omega_{1}=1$ we can set $D_{j, 10}=0$ in (14)

$$
D_{i, 10}=D^{*}-\theta \pi q\left[\Lambda\left(\frac{D_{i, 10}}{D^{*}}\right)^{\frac{1}{\theta}}-1\right] ;
$$

imposing $D_{i, 10}=D^{*}$, and solving for $\Lambda$ we obtain

$$
\Lambda_{10}^{*}=1,
$$

which together with $d D_{10} / d \Lambda<0$ for $\Lambda>0$, delivers the results of part (ii) for $w(t)=\omega_{1}=1$.

\section{Proposition 3}

Proof. Part (i). With $\omega_{0}<1$ and $\omega_{1}<1$, from (11) we know that $D_{j, 11}$ is strictly positive and independent of $\omega_{1}: D_{j, 11}=D^{*} /(2-\theta)$. Calculating the total differential of $(19)$ for a change in $\Omega$, and rearranging we obtain that

$$
\left[1+\frac{\pi p \Omega^{-1}}{D_{j, 01}}\left(\frac{D_{j, 01}}{D^{*} /(2-\theta)}\right)^{\frac{1}{\theta}}\right] \frac{d D_{j, 01}}{d \Omega}-\theta \pi p \Omega^{-2}\left(\frac{D_{j, 01}}{D^{*} /(2-\theta)}\right)^{\frac{1}{\theta}}=(\theta-1) \frac{d D_{i, 01}}{d \Omega} .
$$

Similarly, from the total differential of (18) we get

$$
\left[1+\frac{\pi p}{D_{i, 01}}\left(\frac{D_{i, 01}}{D^{*} /(2-\theta)}\right)^{\frac{1}{\theta}}\right] \frac{d D_{i, 01}}{d \Omega}=(\theta-1) \frac{d D_{j, 01}}{d \Omega}
$$

Plugging (21) into [20), and solving for $d D_{j, 01} / d \Omega$

$$
\frac{d D_{j, 01}}{d \Omega}=\frac{\theta \pi p \Omega^{-2}\left(\frac{D_{j, 01}}{D^{*} /(2-\theta)}\right)^{\frac{1}{\theta}}}{1+\frac{\pi p \Omega^{-1}}{D_{j, 01}}\left(\frac{D_{j, 01}}{D^{*} /(2-\theta)}\right)^{\frac{1}{\theta}}-(\theta-1)^{2}\left[1+\frac{\pi p}{D_{i, 01}}\left(\frac{D_{i, 01}}{D^{*} /(2-\theta)}\right)^{\frac{1}{\theta}}\right]^{-1}}>0,
$$

where the sign of this expression follows from

$$
(\theta-1)^{2}\left[1+\frac{\pi p}{D_{i, 01}}\left(\frac{D_{i, 01}}{D^{*} /(2-\theta)}\right)^{\frac{1}{\theta}}\right]^{-1}<1
$$

The latter holds for any $\theta \in(1,2)$. From 21 and $\frac{d D_{j, 01}}{d \Omega}>0$ it follows that $\frac{d D_{i, 01}}{d \Omega}>0$. Hence, $\frac{d D_{01}}{d \Omega}>0$ for any $\Omega \in(0, \infty)$. 
Part (ii). With $\omega_{1}<\omega_{0}=1$ we have that $D_{j, 01}=0$, and thus from (18), the best-response by the owner, $\left.D_{i, 01}\right|_{\omega_{0}=1}$, is implicitly given by

$$
\left.D_{i, 01}\right|_{\omega_{0}=1}+\theta \pi p\left[(2-\theta) \frac{\left.D_{i, 01}\right|_{\omega_{0}=1}}{D^{*}}\right]^{\frac{1}{\theta}}=D^{*}+\theta \pi p .
$$

If we set $D_{i, 01}=D^{*}$ the above condition is violated, with the right hand side strictly greater than the left hand side. Given that the latter is strictly increasing in $D_{i, 01}$, the solution must be given by $\left.D_{i, 01}\right|_{\omega_{0}=1}>D^{*}$.

With $\omega_{0}<1$ and $\omega_{1}<1$ we must have $D_{j, 01}>0$, and rewriting (18) the solution to $D_{i, 01}$ is implicitly given by

$$
D_{i, 01}+\theta \pi p\left[(2-\theta) \frac{D_{i, 01}}{D^{*}}\right]^{\frac{1}{\theta}}=D^{*}+(\theta-1) D_{j, 01}+\theta \pi p,
$$

which by $\theta \in(1,2)$, and $D_{j, 01}>0$ implies $D_{i, 01}>\left.D_{i, 01}\right|_{\omega_{0}=1}>D^{*}$.

Finally, with $\omega_{0}<\omega_{1}=1$, we have $D_{j, 01}>0$ and $D_{i, 11}=D^{*}$. Using this in 18

$$
\left.D_{i, 01}\right|_{\omega_{1}=1}+\theta \pi p\left(\frac{\left.D_{i, 01}\right|_{\omega_{1}=1}}{D^{*}}\right)^{\frac{1}{\theta}}=D^{*}+(\theta-1) D_{j, 01}+\theta \pi p .
$$

Setting $D_{i, 01}=D^{*}$ the above condition is violated, with the right hand side being strictly greater than the left hand side. Given that the latter is strictly increasing in $D_{i, 01}$, the solution must be given by $\left.D_{i, 01}\right|_{\omega_{1}=1}>D^{*}$.

Thus, regardless of the level of wealth protection in regime 01 , and the subsequent regime 11, $D_{i, 01}>D^{*}$ and therefore total depletion, $D_{01}$ will be above the optimal level $D^{*}$

\section{Proposition 4}

Proof. Part (i). Let us first establish that $d D_{00} / d \Lambda \leq 0$, irrespective of the current and future level of wealth protection.

With $\omega_{0}<1$ and $\omega_{1}<1$, using (8) in (6) and (9) in (7), taking the total differential of these expressions, for a change in $\Lambda$, and rearranging we obtain:

$$
\begin{gathered}
M_{i} \frac{d D_{i, 00}}{d \Lambda}=(\theta-1) \frac{d D_{j, 00}}{d \Lambda}+\pi p(1-q) \frac{D_{i, 00}^{\frac{1}{\theta}}}{D_{i, 10}^{\frac{1}{\theta}+1}} \frac{d D_{i, 10}}{d \Lambda}-N_{i}, \\
M_{j} \frac{d D_{j, 00}}{d \Lambda}=(\theta-1) \frac{d D_{i, 00}}{d \Lambda}+\pi p(1-q) \Omega^{-1} \frac{D_{j, 00}^{\frac{1}{\theta}}}{D_{j, 10}^{\frac{1}{\theta}+1}} \frac{d D_{j, 10}}{d \Lambda}-N_{j} ;
\end{gathered}
$$


where

$$
M_{i} \equiv 1+\sum_{\tilde{x} \tilde{y} \neq x y} \pi_{00 \rightarrow \tilde{x} \tilde{y}} \frac{\lambda_{\tilde{y}}^{2}}{\lambda_{y}^{2}} \frac{D_{i, 00}^{\frac{1}{\theta}-1}}{D_{i, \tilde{x} \tilde{y}}^{\frac{1}{\theta}}}>1 ; M_{j} \equiv 1+\sum_{\tilde{x} \tilde{y} \neq x y} \pi_{00 \rightarrow \tilde{x} \tilde{y}} \frac{\left(1-\omega_{\tilde{x}}\right) \lambda_{\tilde{y}}^{2}}{\left(1-\omega_{0}\right) \lambda_{0}^{2}} \frac{D_{j, 00}^{\frac{1}{\theta}-1}}{D_{j, \tilde{x} \tilde{y}}^{\frac{1}{\theta}}}>1,
$$

and

$$
N_{i} \equiv 2 \theta \pi q \Lambda D_{i, 00}^{\frac{1}{\theta}}\left[\frac{(1-p)}{D_{i, 01}^{\frac{1}{\theta}}}+\frac{p}{D_{i, 11}^{\frac{1}{\theta}}}\right]>0 ; N_{j} \equiv 2 \theta \pi q \Lambda D_{j, 00}^{\frac{1}{\theta}}\left[\frac{(1-p)}{D_{j, 01}^{\frac{1}{\theta}}}+\Omega^{-1} \frac{p}{D_{i, 11}^{\frac{1}{\theta}}}\right]>0 .
$$

Plugging (23) into (22) and solving for $d D_{i, 00} / d \Lambda$

$$
\frac{d D_{i, 00}}{d \Lambda}=\frac{\left(\frac{\theta-1}{M_{j}}\right)\left[\pi p(1-q) \Omega^{-1} \frac{D_{j, 00}^{\frac{1}{\theta}}}{D_{j, 10}^{\frac{1}{\theta}+1}} \frac{d D_{j, 10}}{d \Lambda}-N_{j}\right]+\pi p(1-q) \frac{D_{i, 00}^{\frac{1}{\theta}}}{D_{i, 10}^{\frac{1}{\theta}+1}} \frac{d D_{i, 10}}{d \Lambda}-N_{i}}{M_{i}-\frac{(\theta-1)^{2}}{M_{j}}} \leq 0,
$$

with strict inequality for $\Lambda>0$.

Given $D_{i, 10}, D_{j, 10} \geq 0$, as established by lemma 1 and $d D_{i, 10} / d \Lambda, d D_{j, 10} / d \Lambda \leq 0$ as established by Proposition 2. the sign of $d D_{i, 00} / d \Lambda$ follows from $M_{i}>1>(\theta-1)^{2} M_{j}^{-1}$, which holds for any $\theta \in$ $(1,2)$. Back into 23), $d D_{i, 00} / d \Lambda \leq 0$ implies $d D_{j, 00} / d \Lambda \leq 0$ with strict inequality for $\Lambda>0$. Thus, $d D_{00} / d \Lambda \leq 0$, with strict inequality for $\Lambda>0$.

With $\omega_{0}<\omega_{1}=1$, we have that $D_{j, 10}=D_{j, 11}=0$. Hence 23) simplifies to

$$
\tilde{M}_{j} \frac{d D_{j, 00}}{d \Lambda}=(\theta-1) \frac{d D_{i, 00}}{d \Lambda}-2 \theta \pi(1-p) q \Lambda\left(\frac{D_{j, 00}}{D_{j, 01}}\right)^{\frac{1}{\theta}}
$$

with

$$
\tilde{M}_{j} \equiv 1+\pi(1-p) q \Lambda^{2} \frac{D_{j, 00}^{\frac{1}{\theta}-1}}{D_{j, 01}^{\frac{1}{\theta}}}>1 .
$$

Using this in (22) and solving for $d D_{i, 00} / d \Lambda$

$$
\frac{d D_{i, 00}}{d \Lambda}=\frac{\frac{(\theta-1)}{\tilde{M}_{j}}\left[-2 \theta \pi \Lambda(1-p) q\left(\frac{D_{j, 00}}{D_{j, 01}}\right)^{\frac{1}{\theta}}\right]+\pi p(1-q) \frac{D_{i, 00}^{\frac{1}{\theta}}}{D_{i, 10}^{\frac{1}{\theta}+1}} \frac{d D_{i, 10}}{d \Lambda}-N_{i}}{M_{i}-\frac{(\theta-1)^{2}}{\tilde{M}_{j}}} \leq 0,
$$

with strict inequality for $\Lambda>0$. Given $d D_{i, 10} / d \Lambda \leq 0$ as established by Proposition 2 the sign of this expression immediately follows from $M_{i}>1>(\theta-1)^{2} \tilde{M}_{j}^{-1}$, which holds for any $\theta \in(1,2)$. Back into (26), $d D_{i, 00} / d \Lambda \leq 0$ implies $d D_{j, 00} / d \Lambda \leq 0$, with strict inequality for $\Lambda>0$. Thus, $d D_{00} / d \Lambda \leq 0$, with strict inequality for $\Lambda>0$.

With $\omega_{1}<\omega_{0}=1$ and thus $D_{j, 00}=0$, we can solely focus on the effect of $\Lambda$ on $D_{i, 00}$. In this case 
the (22) simplifies to

$$
M_{i} \frac{d D_{i, 00}}{d \Lambda}=\pi p(1-q) \frac{D_{i, 00}^{\frac{1}{\theta}}}{D_{i, 10}^{\frac{1}{\theta}+1}} \frac{d D_{i, 10}}{d \Lambda}-N_{i} .
$$

Given that $M_{i}>1, N_{i}>0$, and that $d D_{i, 10} / d \Lambda \leq 0$ as established by Proposition 2, it follows that $d D_{i, 00} / d \Lambda \leq 0$ and therefore $d D_{00} / d \Lambda \leq 0$, with strict inequality for $\Lambda>0$.

Finally, note that $\omega_{0}=\omega_{1}$ would imply static institutions along the wealth regime, and hence regime 00 collapses to regime 10, where the results from Proposition 2 follow.

Part (ii). Now that we have established that that $d D_{00} / d \Lambda \leq 0$, irrespective of the current and future level of wealth protection, we proceed to show the existence of the $\Lambda_{00}^{*}(\Omega)$ threshold. For this, first note that $\lambda_{0}=0$ implies $D_{00}=0$. With dynamic institutions, i.e., $\lambda_{1} \neq \lambda_{0}$, this translates into $\lim _{\Lambda \rightarrow \infty} D_{00}=0$. In turn, note that with $\Lambda=1 \leftrightarrow \lambda_{0}=\lambda_{1}$, regime 00 is equivalent to regime 01 . Using the results for regime 01 in Proposition 3 we know that $D_{00}>D^{*}$ if $\Lambda=1$. Using $d D_{00} / d \Lambda<0$ for $\Lambda>0, \lim _{\Lambda \rightarrow \infty} D_{00}=0, D_{00}>D^{*}$ if $\Lambda=1$, and the intermediate value theorem, there must exist a unique value $\Lambda_{00}^{*}(\Omega)>1$ such that the conditions of Proposition 4 hold.

\section{Proposition 5}

Proof. With $\omega_{1}<1$, using (8) in (6) and (9) in (7), taking the total differential of these expressions, for a change in $\Omega$, and rearranging we obtain

$$
\begin{gathered}
M_{i} \frac{d D_{i, 00}}{d \Omega}=(\theta-1) \frac{d D_{j, 00}}{d \Omega}+\pi(1-p) q \frac{D_{i, 00}^{\frac{1}{\theta}}}{D_{i, 01}^{\frac{1}{\theta}+1}} \frac{d D_{i, 01}}{d \Omega}, \\
M_{j} \frac{d D_{j, 00}}{d \Omega}=(\theta-1) \frac{d D_{i, 00}}{d \Omega}+\pi(1-p) q \Omega^{-1} \frac{D_{j, 00}^{\frac{1}{\theta}}}{D_{j, 01}^{\frac{1}{\theta}+1}} \frac{d D_{j, 01}}{d \Omega}+\tilde{N}_{j} ;
\end{gathered}
$$

with $M_{i}$ and $M_{j}$ as defined in (24) and

$$
\tilde{N}_{j} \equiv \theta \pi \Omega^{-2} D_{j, 00}^{\frac{1}{\theta}}\left[\frac{p(1-q)}{D_{j, 10}^{\frac{1}{\theta}}}+\frac{p q \Lambda^{2}}{D_{j, 11}^{\frac{1}{\theta}}}\right]>0 .
$$

Plugging (27) into (28) and solving for $d D_{j, 00} / d \Omega$

$$
\frac{d D_{j, 00}}{d \Omega}=\frac{(\theta-1) \frac{\pi(1-p) q \frac{D_{i, j 00}^{\frac{1}{\theta}}}{D_{i, 01}^{\theta}} \frac{d D_{i, 01}}{d \Omega}}{M_{i}}+\pi(1-p) q \Omega^{-1} \frac{D_{j, 00}^{\frac{1}{\theta}}}{D_{j, 01}^{\frac{1}{\theta}+1}} \frac{d D_{j, 01}}{d \Omega}+\tilde{N}_{j}}{M_{j}+\frac{(\theta-1)^{2}}{M_{i}}}>0 ;
$$

where the sign follows from $M_{i}>1$ and $M_{j}>1$ as defined in (24), and $d D_{i, 01} / d \Omega>0$ and $d D_{j, 01} / d \Omega>0$ as established in the proof of Proposition 3. In turn, from $d D_{j, 00} / d \Omega>0 d D_{i, 01} / d \Omega>0$, and $M_{i}>1$ in 
27 it follows that $d D_{i, 00} / d \Omega>0$. Therefore $d D_{00} / d \Omega>0$ for any $\Omega \in(0, \infty)$.

\section{A.2 Lemmas}

\section{Lemma 1}

Proof. Let us first establish that, in the $\left(D_{i, x y}, D_{j, x y}\right) \in \mathcal{R}_{+}^{2}$ space, the intercept of the reaction of function of the owner (6) is below the intercept of the reaction function of the trespasser (7). For this, we set $D_{i, x y}=0$, from which we obtain that the intercept of the owner's reaction function (6) with the $D_{i, x y}=0$-axis is $\left.D_{j, x y}^{i}\right|_{D_{i}=0}=-\left(D^{*}-\theta g_{i, x y}\right)(\theta-1)^{-1}$, and strictly negative as $D_{i, x y}=0$ in (8) implies $g_{i, x y}=-\sum_{\tilde{x} \tilde{y} \neq x y} \pi_{x y \rightarrow \tilde{x} \tilde{y}}<0$. Similarly, setting $D_{i, x y}=0$ in the reaction function of the trespasser (7), we obtain $D_{j, x y}+g_{j, x y}=D^{*}$, where the left hand side is equal to $-\sum_{\tilde{x} \tilde{y} \neq x y} \pi_{x y \rightarrow \tilde{x} \tilde{y}}$ when $D_{j, x y}=0$ and is strictly increasing in $D_{j, x y}$. This implies that the intercept of the trespasser's reaction function with the $D_{i, x y}=0$-axis, $\left.D_{j, x y}^{j}\right|_{D_{i}=0^{\prime}}$ is strictly positive.

Now we proceed to establish that, in the $\left(D_{i, x y}, D_{j, x y}\right) \in \mathcal{R}_{+}^{2}$ space, the reaction function of the owner (6) is always stepper than the reaction function of the trespasser (7). Taking the total differential of (6) with respect to $D_{i, x y}$ and rearranging, we get the slope of the owner's reaction function in the $\left(D_{i, x y}, D_{j, x y}\right) \in \mathcal{R}_{+}^{2}$ space:

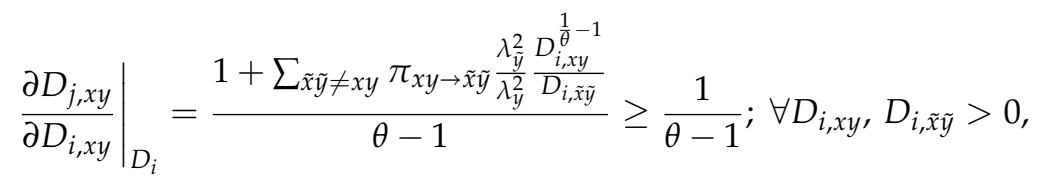

Similarly, the total differential of (7) gives the slope of the trespasser's reaction function in the $\left(D_{i, x y}, D_{j, x y}\right) \in$ $\mathcal{R}_{+}^{2}$ space:

$$
\left.\frac{\partial D_{j, x y}}{\partial D_{i, x y}}\right|_{D_{j}}=\frac{\theta-1}{1+\sum_{\tilde{x} \tilde{y} \neq x y} \pi_{x y \rightarrow \tilde{x} \tilde{y} \tilde{y}} \lambda_{y}^{2} \frac{D_{j, x y}^{\frac{1}{\theta}-1}}{D_{j, \tilde{x} \tilde{y}}}} \leq \theta-1 ; \forall D_{j, x y}, D_{j, \tilde{x} \tilde{y}}>0,
$$

with $\theta \in(1,2)$, and hence $(\theta-1)^{-1}>\theta-1$, from $(29)$ and (30) we obtain that

$$
\left.\frac{\partial D_{j, x y}}{\partial D_{i, x y}}\right|_{D_{i}}>\left.\frac{\partial D_{j, x y}}{\partial D_{i, x y}}\right|_{D_{j}} .
$$

Given that this strict inequality holds for all $\left(D_{i, x y}, D_{j, x y}\right) \in \mathcal{R}_{+}^{2}$ and that $\left.D_{j, x y}^{j}\right|_{D_{i}=0}>0>\left.D_{j, x y}^{i}\right|_{D_{i}=0^{\prime}}$ then the two reaction functions $(6)$ and $(7)$ must have a single crossing point in the $\left(D_{i, x y}, D_{j, x y}\right) \in \mathcal{R}_{+}^{2}$ space. 